\title{
A solution to the bidimensional Global Asymptotic Stability Conjecture
}

by

Carlos GUTIERREZ

IMPA, Rio de Janeiro

ABSTRACT. - If $Y: \mathfrak{R}^{2} \rightarrow \mathfrak{R}^{2}$ is a $C^{1}$ vector field such that $Y(0)=0$ and, for all $q \in \Re^{2}$, all the eigenvalues of $D X(q)$ have negative real part, then the stable manifold of 0 is $\Re^{2}$.

Let $\rho \in[0, \infty)$ and $Y: \mathfrak{R}^{2} \rightarrow \mathfrak{R}^{2}$ be a $C^{1}$ map such that, for all $q \in \mathfrak{R}^{2}$, the determinant of $D Y(q)$ is positive and moreover, for all $p \in \mathfrak{R}^{2}$, with $|p| \geq \rho$, the spectrum of $D Y(p)$ is disjoint of the non-negative real half axis. Then $Y$ is injective.

Key words: Asymptotic stability, Univalent maps.

RÉSUMÉ. - Si $Y: \mathfrak{R}^{2} \rightarrow \mathfrak{R}^{2}$ est un champ de vecteur tel que $Y(0)=0$ et, pour tout $q \in \mathfrak{R}^{2}$, les valeurs propres de $D Y(q)$ ont leur partie réelle négative, alors la variété stable de 0 est $\mathfrak{R}^{2}$.

Soit $\rho \in[0, \infty)$ et $Y: \mathfrak{R}^{2} \rightarrow \mathfrak{R}^{2}$ une application $C^{1}$ telle que, pour tout $q \in \mathfrak{R}^{2}$, le déterminant de $D Y(q)$ est positif et de plus, pour tout $p \in \mathfrak{R}^{2}$, avec $|p| \geq \rho$, le spectre de $D Y(p)$ est disjoint du demi-axe réel non négatif. Alors $Y$ est injective.

\section{INTRODUCTION}

This work is related to the conjecture about global asymptotic stability which claims that if a $C^{1}$ vector field $Y: \mathfrak{R}^{\mathfrak{n}} \rightarrow \mathfrak{R}^{\mathfrak{n}}$ has a singularity $p$

A.M.S. Classification: (34) Ordinary differential equations; (58) Global analysis, analysis on manifolds. 
and, for every $q \in \mathfrak{R}^{\mathfrak{n}}$, all the eigenvalues of $D Y(q)$ have negative real part then the basin of attraction of $p$ is the whole $\mathfrak{R}^{\mathrm{n}}$. This conjecture was explicitly stated by Marcus and Yamabe [12] in 1960. Also, it somehow stems from the Aizerman Problem [10], [1] (1949). Barabanov [2] proved that this conjecture is false if $n \geq 4$. Here we prove that it is true if $n=2$.

In the bidimensional case, the conjecture above has been solved affirmatively under additional conditions since Krasovskii's work [11]. Markus and Yamabe [12] considered the case where one of the partial derivatives of $Y$ vanishes identically. Hartmann [8] solved the problem when $D Y(x)+D Y(x)^{T}$ is everywhere negative definite, where $T$ means transposition. Olech [15] proved the conjecture when there exist constants $\delta>0$ and $R>0$ such that $|x|>R$ implies that $|Y(x)|>\delta$. By the work of Meisters and Olech, the conjecture was known to be true for polynomial vector fields [14]. The fact that the assumptions in the conjecture are to be global is completely justified by the examples of [7].

In higher dimensions and under additional hypotheses, there are also positive answers. There is a rich literature on the subject; we suggest the reader [13] for further references and history of the problem. Also Hartman's book [9] deals with this question. Concerning some recent previous results we wish to mention the works of Gasull, Llibre and Sotomayor [4], Gasull and Sotomayor [5], and Gorni and Zampieri [6].

Let us proceed to state, in a more precise way, the results of this paper.

Let $\rho \in[0, \infty)$ and let $Y: \mathfrak{R}^{2} \rightarrow \mathfrak{R}^{2}$ be a map of class $C^{1}$. We say that $Y$ satisfies the $\rho$-eigenvalue condition if, for all $q \in \mathfrak{R}^{2}$, the determinant of $D Y(q)$ is positive and moreover, for all $p \in \mathfrak{R}^{2}$, with $|p| \geq \rho$, the spectrum of $D Y(p)$ is disjoint of the non-negative real half axis.

THEOREM A. - If $Y: \mathfrak{R}^{2} \rightarrow \mathfrak{R}^{2}$ is a map of class $C^{1}$ that satisfies the $\rho$-eigenvalue condition, for some $\rho \in[0, \infty)$, then $Y$ is injective.

THEOREM B. - Let $Y: \mathfrak{R}^{2} \rightarrow \mathfrak{R}^{2}$ be a vector field of class $C^{1}$ such that, for all $q \in \mathfrak{R}^{2}$, all the eigenvalues of $D X(q)$ have negative real part. If $Y$ has a singularity, say $p$, then the stable manifold of $p$ is $\mathfrak{R}^{2}$.

Theorem B is the solution to the Global Asymptotic Stability Conjecture.

A very interesting results of injectivity of maps, based on spectral conditions too, have been found by B. Smith and F. Xavier [16].

In Theorem $\mathrm{A}$, we shall assume that $Y$ is at least $C^{2}$. It is enough to prove it under these conditions. In fact, first observe that, by the assumptions and the Inverse Mapping Theorem, non-injectivity is an open condition. Therefore, if there was a non-injective $C^{1}$ map satisfying the $\rho$-eigenvalue condition, it could be approximated (in the Whitney topology) by a smooth map that would have both of these two properties. 
Theorem B is a direct consequence of Theorem A and the Olech's Theorem [15] that states that if the assumptions in Theorem B imply that $Y$ is injective, then the conclusion of Theorem B is true.

This work contains two more important results, Theorems $\mathrm{C}$ and $\mathrm{D}$, stated at the beginning of Section 2 below. I wish to thank J. Llibre, A. Gasull and M. Wattenberg for very important comments. I wish also to thank A. Katok, R. de la Llave, C. Pugh and R. Roussarie for very helpful conversations. Sometime after the present work was completed, I took knowledge that Robert Fessler [3] was obtaining similar results.

\section{THE HALF-REEB COMPONENTS}

Let $\rho \in[0, \infty)$. Any map $Y=(f, g)$, as above, satisfying the $\rho$-eigenvalue condition, possesses the $\rho$-obstruction property: For all $(x, y) \in \mathfrak{R}^{2}$, with $|(x, y)| \geq \rho$,

$$
\nabla f(x, y) \notin(0, \infty) \times\{0\} .
$$

Given a smooth regular curve $\gamma:[a, b] \rightarrow \mathfrak{R}^{2}$ (resp. a smooth function $f: \mathfrak{R}^{2} \rightarrow \mathfrak{R}$ ) and $t \in[a, b]$ (resp. $q \in \mathfrak{R}^{2}$ ), we shall denote by $\gamma^{\prime}(t)^{\perp}$ (resp. by $\nabla f^{\perp}(q)$ ) the vector field of $\mathfrak{R}^{2}$ having the same length as $\gamma^{\prime}(t)$ (resp. $\nabla f(p)$ ), being orthogonal to $\gamma^{\prime}(t)$ (resp. $\nabla f(p)$ ) and such that $\left\{\gamma^{\prime}(t), \gamma^{\prime}(t)^{\perp}\right\}$ (resp. $\left.\left\{\nabla f(p), \nabla f^{\perp}(p)\right\}\right)$ is a positive basis of $\mathfrak{R}^{2}$.

Let $D$ be a smooth bidimensional submanifold of $\Re^{2}$ with boundary and, possibly, corner $\partial D$. Let $\gamma:[a, b] \rightarrow \partial D$ be a smooth regular curve. We say that $D$ is on the left, or more precisely, on the left side of $\gamma$ (resp. on the right of $\gamma)$, if for all $s \in(a, b)$, or equivalently for some $s \in(a, b)$, $\gamma^{\prime}(s)^{\perp}$ points inward $D$ (resp. $\gamma^{\prime}(s)^{\perp}$ points outward $D$ ).

Throughout this work, $X=(f, g): \mathfrak{R}^{2} \rightarrow \mathfrak{R}^{2}$ will denote a $C^{2}$ map satisfying the $\rho$-eigenvalue condition, for some $\rho \in[0, \infty)$. Given $p \in \mathfrak{R}^{2}$, denote by $F_{p}=F_{p}(t), t \in \mathfrak{R}$, the trajectory of $\nabla f^{\perp}$ such that $F_{p}(0)=p$. Also, $F_{p}^{+}=F_{p}^{+}(t)$ and $F_{p}^{-}=F_{p}^{-}(t), t \in \mathfrak{R}$, will denote the positive and negative half-trajectories of $\nabla f^{\perp}$, respectively, starting at $p$.

Given $\theta \in \mathfrak{R}$, denote by

$$
H_{\theta}=\left(\begin{array}{cc}
\cos \theta & -\sin \theta \\
\sin \theta & \cos \theta
\end{array}\right)
$$

In this way

$$
H_{\theta}^{-1}=\left(\begin{array}{cc}
\cos \theta & \sin \theta \\
-\sin \theta & \cos \theta
\end{array}\right)
$$

Vol. $12, \mathrm{n}^{\circ}$ 6-1995. 
Let $X_{\theta}=H_{\theta} \circ X \circ H_{\theta}^{-1}=\left(f_{\theta}, g_{\theta}\right)$.

Remark. - For all $\theta \in \mathfrak{R}, X_{\theta}$ satisfies the $\rho$-eigenvalue condition and therefore the $\rho$-obstruction property.

In fact, this follows immediately from:

$$
D X_{\theta}(x, y)=H_{\theta} \circ D X_{H_{\theta}^{-1}(x, y)} \circ H_{\theta}^{-1} \text {. }
$$

It will be seen that the proof of Theorem A, depends only on the Remark above and on the fact that $D X(p)$ is invertible, for all $p \in \mathfrak{R}^{2}$. For this reason, it will become clear that the arguments of this work apply, almost word for word, to give the following more general result:

ThEOREM C. - Let $Y: \mathfrak{R}^{2} \rightarrow \mathfrak{R}^{2}$ be a $C^{1}$ map such that, for all $p \in \mathfrak{R}^{2}$, $D Y(p)$ is invertible. Suppose that there exists $v \in \mathfrak{R}^{2}$, with $\|v\|=1$, such that the following (directional) obstruction property is satisfied: For all $\theta \in \mathfrak{R}$ and for all $p \in \mathfrak{R}^{2}$, with $|p| \geq \rho$,

$$
\nabla \tilde{f}_{\theta}(p) \cdot v \neq\left\|\nabla \tilde{f}_{\theta}(p)\right\|
$$

where, $\left(\tilde{f}_{\theta}, \tilde{g}_{\theta}\right)=H_{\theta} \circ Y \circ H_{\theta}^{-1}$.

Then, $Y$ is injective.

Theorem $\mathrm{A}$ is a particular case of Theorem $\mathrm{C}$ because if $v=(1,0)$ in Theorem $\mathrm{C}$, then (for all $\theta \in \mathfrak{R}$ ) $Y_{\theta}$ satisfies the $\rho$-obstruction property.

Given $p, q \in \mathfrak{R}^{2},[p, q]$ will denote a non-necessarily oriented segment connecting $p$ and $q$. If $\gamma$ is a curve and $p, q \in \gamma,[p, q]_{\gamma}$ (resp. $\left.(p, q)_{\gamma}\right)$ will denote the closed (resp. open) subinterval of $\gamma$ with endpoints $p$ and $q$. The notation $[p, q]_{f_{\theta}}$ and $(p, q)_{f_{\theta}}$ will refer to arcs of trajectory of $\nabla f_{\theta}^{\perp}$.

Let $\mu:(0,2) \rightarrow \mathfrak{R}$ be a smooth map with $\mu^{\prime}(1)=\mu(1)=0, \mu^{\prime \prime}(1)<0$ and $\mu^{\prime}(x) \neq 0$ for all $x \neq 1$ (in particular, 1 is the absolute maximum value of $\mu$ ). Suppose also that $\lim _{x \rightarrow 0} \mu(x)=\lim _{x \rightarrow 2} \mu(x)=-\infty$. Let $\mathcal{G}$ be the foliation on $[0,2] \times \mathfrak{R}$ whose leaves are $\{0\} \times \mathfrak{R},\{2\} \times \mathfrak{R}$ and the family of the graphs of the functions $\mu+c$, where $c \in \mathfrak{R}$. This foliation $\mathcal{G}$ is usually known as a Reeb component. We say that $A \subset \mathfrak{R}^{2}$ is a Half-Reeb component of $\nabla f^{\perp}$ if the foliation on $A$ determined by the trajectories of $\nabla f^{\perp}$ is topologically equivalent to the foliation $\mathcal{G}$ (just defined above) restricted to the manifold with boundary and corner $[0,2] \times[0, \infty)$ in such a way that if

$$
h: \quad[0,2] \times[0, \infty] \rightarrow A
$$

is the homeomorphism producing the topological equivalence and if $p_{i}=h((i, 0))$, then $\left[p_{0}, p_{2}\right]=h([0,2] \times\{0\})$ is a smooth segment 
transversal to $\nabla f^{\perp}$ in the complement of $\left\{p_{1}\right\}$ and also, for some $\delta \in\{-,+\}, F_{p_{0}}^{\delta}=h(\{0\} \times[0, \infty))$ and $F_{p_{2}}^{-\delta}=h(\{2\} \times[0, \infty))$. See fig. 2.1.

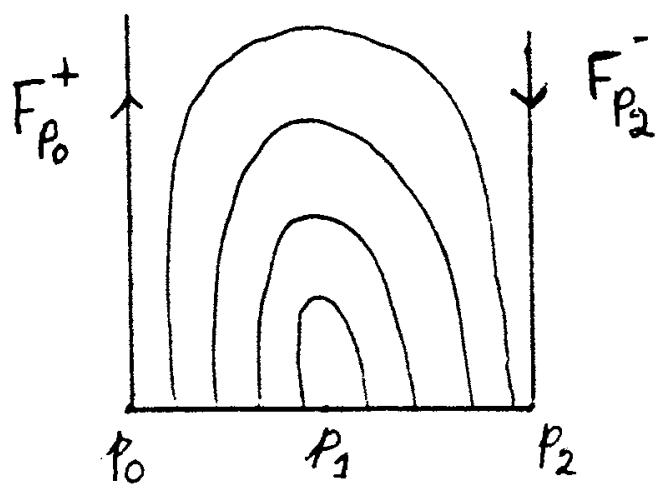

Fig. 2.1

Denote by $\partial_{1} A$ the union of the half-trajectories $F_{p_{0}}^{\delta}$ and $F_{p_{2}}^{-\delta}$ and the segment $\left[p_{0}, p_{2}\right]$ which will be called edges of $A$. Observe that $A$ may not be a closed subset of $\mathfrak{R}^{2}$.

The main result of this section is the following

PROPOSITION 2.1. - There is no smooth regular curve $\gamma:[0,3] \rightarrow \mathfrak{R}^{2}$ such that the following properties are satisfied:

(a) For some $1<s \leq t<2, \gamma$ is transversal to $\nabla f^{\perp}$ at all the points of $[0,1] \cup[s, t] \cup[2,3]$.

(b) $\gamma([1, s])$ and $\gamma([t, 2])$ are the compact edges of two half-Reeb components of $\nabla f^{\perp}$ which are both either on the left of $\gamma$ or on the right of $\gamma$.

See in figure 2.2 the situation that cannot occur.

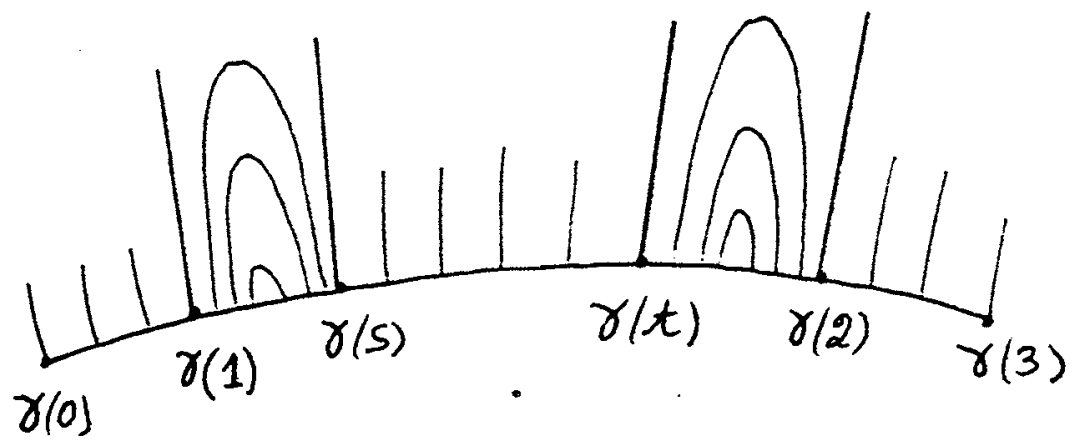

FIG. 2.2

Vol. 12, $n^{\circ}$ 6-1995. 
The $\rho$-obstruction property is only used to prove Proposition 2.1 . For this reason, it will become clear that the arguments of this work apply, word for word, to give the following more general result:

THEOREM D. - Let $Y: \mathfrak{R}^{2} \rightarrow \mathfrak{R}^{2}$ be a $C^{1}$ map such that, for all $p \in \mathfrak{R}^{2}$, $D Y(p)$ is invertible and, for all $\theta \in \mathfrak{R}, \nabla \tilde{f}_{\theta}^{\perp}$ satisfies the same properties as $\nabla f^{\perp}$ does in Proposition 2.1, where $Y_{\theta}=H_{\theta} \circ Y \circ H_{\theta}^{-1}=\left(\tilde{f}_{\theta}, \tilde{g}_{\theta}\right)$.Then, $Y$ is injective.

To prove Proposition 2.1 we shall need Lemmas 2.2-2.6.

LEMMA 2.2. - (a) For all $p \in \mathfrak{R}^{2}$, the function

$$
\varphi(\theta)=\operatorname{det}\left(H_{\theta}\left(\nabla f^{\perp}(p)\right), \nabla f_{\theta}^{\perp}\left(H_{\theta}(p)\right)\right)
$$

satisfies $\varphi(0)=0$ and $\varphi^{\prime}(0)=-\operatorname{det}(D X(p))$, where $\left(H_{\theta}\left(\nabla f^{\perp}(p)\right)\right.$ and $\nabla f_{\theta}^{\perp}\left(H_{\theta}(p)\right)$ are to be the first and second column vector of the considered matrix. In other words, for small $\theta>0$ fixed, the speed vector $\left(H_{\theta}\left(\nabla f^{\perp}(p)\right)\right.$ of the curve $t \rightarrow H_{\theta}\left(F_{p}^{+}(t)\right)$, at the point $H_{\theta}(p)$, and $\nabla f_{\theta}^{\perp}\left(H_{\theta}(p)\right)$ form a negative basis.

(b) Let $p \in \mathfrak{R}^{2}$ and $0<\theta<\pi$ be fixed but arbitrary. The level curves of $f_{\theta}$ are transversal to $H_{\theta}\left(F_{p}\right)$.

Proof. - Given $v=\left(v_{1}, v_{2}\right), w=\left(w_{1}, w_{2}\right) \in \mathfrak{R}^{2}$, we shall denote by

$$
\begin{aligned}
& (v, w)=\left(\begin{array}{ll}
v_{1} & w_{1} \\
v_{2} & w_{2}
\end{array}\right) \\
& \left(\begin{array}{c}
v \\
w
\end{array}\right)=\left(\begin{array}{ll}
v_{1} & v_{2} \\
w_{1} & w_{2}
\end{array}\right)
\end{aligned}
$$

Recall also that $v^{\perp}=\left(-v_{2}, v_{1}\right)$. The following holds:

$$
\left\{\begin{array}{l}
\left(\begin{array}{c}
v^{\perp} \\
w^{\perp}
\end{array}\right)=\left(\begin{array}{c}
v \\
w
\end{array}\right)\left(\begin{array}{cc}
0 & 1 \\
-1 & 0
\end{array}\right) \\
\left(\begin{array}{c}
-v \\
-w
\end{array}\right)=\left(\begin{array}{c}
v^{\perp} \\
w^{\perp}
\end{array}\right)\left(\begin{array}{cc}
0 & 1 \\
-1 & 0
\end{array}\right) \\
\left(\begin{array}{c}
-w \\
v
\end{array}\right)=\left(\begin{array}{cc}
0 & -1 \\
1 & 0
\end{array}\right)\left(\begin{array}{c}
v \\
w
\end{array}\right)
\end{array}\right.
$$

Observe that

$$
\left\{\begin{array}{l}
\left.\frac{d}{d \theta} H_{\theta}\right|_{\theta=0}=\left(\begin{array}{cc}
0 & -1 \\
1 & 0
\end{array}\right) \\
\text { and } \\
\left.\frac{d}{d \theta} H_{\theta}^{-1}\right|_{\theta=0}=\left(\begin{array}{cc}
0 & 1 \\
-1 & 0
\end{array}\right)
\end{array}\right.
$$


As

$$
\begin{aligned}
\left.\frac{d}{d \theta}\left(H_{\theta}\left(\nabla f^{\perp}(x, y)\right)\right)\right|_{\theta=0} & =\left(\frac{d}{d \theta} H_{\theta}\right)\left(\nabla f^{\perp}(x, y)\right) \\
& =\left(\begin{array}{cc}
0 & -1 \\
1 & 0
\end{array}\right)\left(\nabla f^{\perp}(x, y)\right) \\
& =-\nabla f(x, y)
\end{aligned}
$$

We have that

$$
\left.\frac{d}{d \theta}\left(H_{\theta}\left(\nabla f^{\perp}(x, y)\right)\right)\right|_{\theta=0}=-\nabla f(x, y)
$$

Moreover, the expression

$$
D X_{\theta}(x, y)=H_{\theta} \circ D X_{H_{\theta}^{-1}(x, y)} \circ H_{\theta}^{-1}
$$

can be rewritten, for all $(x, y) \in \mathfrak{R}^{2}$, as

$$
\left(\begin{array}{c}
\nabla f_{\theta}(x, y) \\
\nabla g_{\theta}(x, y)
\end{array}\right)=H_{\theta} \cdot\left(\begin{array}{c}
\nabla f\left(H_{\theta}^{-1}(x, y)\right) \\
\nabla g\left(H_{\theta}^{-1}(x, y)\right)
\end{array}\right) \cdot H_{\theta}^{-1}
$$

Which implies that, for all $(x, y) \in \mathfrak{R}^{2}$,

$$
\left(\begin{array}{c}
\nabla f_{\theta}\left(H_{\theta}(x, y)\right) \\
\nabla g_{\theta}\left(H_{\theta}(x, y)\right)
\end{array}\right)=H_{\theta} \cdot\left(\begin{array}{c}
\nabla f((x, y)) \\
\nabla g((x, y))
\end{array}\right) \cdot H_{\theta}^{-1}
$$

and so (by (1)) that, for all $(x, y) \in \mathfrak{R}^{2}$,

$$
\left(\begin{array}{c}
\nabla f_{\theta}^{\perp}\left(H_{\theta}(x, y)\right) \\
\nabla g_{\theta}^{\perp}\left(H_{\theta}(x, y)\right.
\end{array}\right)=H_{\theta} \cdot\left(\begin{array}{c}
\nabla f(x, y) \\
\nabla g(x, y)
\end{array}\right) \cdot H_{\theta}^{-1} \cdot\left(\begin{array}{cc}
0 & 1 \\
-1 & 0
\end{array}\right)
$$

Hence, by (2),

$$
\begin{aligned}
& \frac{d}{d \theta} \cdot\left.\left(\begin{array}{l}
\nabla f_{\theta}^{\perp}\left(H_{\theta}(x, y)\right) \\
\nabla g_{\theta}^{\perp}\left(H_{\theta}(x, y)\right)
\end{array}\right)\right|_{\theta=0} \\
&=\left(\begin{array}{cc}
0 & -1 \\
1 & 0
\end{array}\right)\left(\begin{array}{l}
\nabla f(x, y) \\
\nabla g(x, y)
\end{array}\right)\left(\begin{array}{cc}
1 & 0 \\
0 & 1
\end{array}\right)\left(\begin{array}{cc}
0 & 1 \\
-1 & 0
\end{array}\right) \\
& \quad \quad+\left(\begin{array}{ll}
1 & 0 \\
0 & 1
\end{array}\right)\left(\begin{array}{l}
\nabla f(x, y) \\
\nabla g(x, y)
\end{array}\right)\left(\begin{array}{cc}
0 & 1 \\
-1 & 0
\end{array}\right)\left(\begin{array}{cc}
0 & 1 \\
-1 & 0
\end{array}\right) \\
&=\left(\begin{array}{cc}
0 & -1 \\
1 & 0
\end{array}\right)\left(\begin{array}{l}
\nabla f^{\perp}(x, y) \\
\nabla g^{\perp}(x, y)
\end{array}\right)+\left(\begin{array}{ll}
\nabla f^{\perp}(x, y) \\
\nabla g^{\perp}(x, y)
\end{array}\right)\left(\begin{array}{cc}
0 & 1 \\
-1 & 0
\end{array}\right) \\
&=\left(\begin{array}{c}
-\nabla g^{\perp}(x, y) \\
\nabla f^{\perp}(x, y)+\left(\begin{array}{l}
-\nabla f(x, y) \\
-\nabla g(x, y)
\end{array}\right) \\
=
\end{array}\right. \\
&\left(\begin{array}{c}
-\nabla g^{\perp}(x, y)-\nabla f(x, y) \\
\nabla f^{\perp}(x, y)-\nabla g(x, y)
\end{array}\right)
\end{aligned}
$$


That is,

$$
\left.\frac{d}{d \theta}\left(\nabla f_{\theta}^{\perp}\left(H_{\theta}(x, y)\right)\right)\right|_{\theta=0}=-\nabla g^{\perp}(x, y)-\nabla f(x, y)
$$

Therefore, using (3),

$$
\begin{aligned}
\frac{d}{d \theta}( & \left.\operatorname{det}\left(H_{\theta}\left(\nabla f^{\perp}(x, y)\right), \nabla f_{\theta}^{\perp}\left(H_{\theta}(x, y)\right)\right)\right)\left.\right|_{\theta=0} \\
= & \operatorname{det}\left(\nabla f^{\perp}(x, y),-\nabla f(x, y)-\nabla g^{\perp}(x, y)\right) \\
& +\operatorname{det}\left(-\nabla f(x, y), \nabla f^{\perp}(x, y)\right) \\
= & \operatorname{det}\left(\nabla f^{\perp}(x, y),-\nabla f(x, y)-\nabla g^{\perp}(x, y)\right) \\
& +\operatorname{det}\left(\nabla f^{\perp}(x, y), \nabla f(x, y)\right) \\
= & \operatorname{det}\left(\nabla f^{\perp}(x, y),-\nabla g^{\perp}(x, y)\right) \\
= & -\operatorname{det}\left[\left(\begin{array}{ll}
\nabla f^{\perp}(x, y) \\
\nabla g^{\perp}(x, y)
\end{array}\right)\left(\begin{array}{cc}
0 & 1 \\
-1 & 0
\end{array}\right)\right] \\
= & -\operatorname{det}\left(\begin{array}{ll}
\nabla f(x, y) \\
\nabla g(x, y)
\end{array}\right)=-\operatorname{det}(D X(x, y))<0 .
\end{aligned}
$$

The proof of (a) of this lemma is finished. Now we shall prove $(b)$. Let $p \in \mathfrak{R}^{2}$ and $\theta \in(0, \pi)$ fixed but arbitrary. Let $\lambda: \mathfrak{R} \rightarrow F_{p}$ be a regular global parametrization of $F_{p}$. By assumption, for all $t \in \mathfrak{R}$, $f(\lambda(t)) \equiv f(p)$; i.e.,

(6) For all $t \in \mathfrak{R}$, the first coordinate of $D X(\lambda(t)) \cdot\left(\lambda^{\prime}(t)\right)$, namely $d f(\lambda(t)) \cdot\left(\lambda^{\prime}(t)\right)$, is zero.

Therefore as, for all $q \in \Re^{2}, D X(q)$ is invertible,

(7) For all $t \in \mathfrak{R}^{2}, d g(\lambda(t)) \cdot\left(\lambda^{\prime}(t)\right)$, is different from zero

It is easy to see that

$$
f_{\theta}\left(H_{\theta}(\lambda(t))\right)=\cos (\theta) \cdot f(\lambda(t))-\sin (\theta) \cdot g(\lambda(t)) .
$$

Item $(b)$ of this lemma follows easily from this expression, (6) and (7) above.

LEMMA 2.3. - Let $C$ be a simple closed continuous curve made up of an arc of trajectory $[p, q]_{f}$ of $\nabla f^{\perp}$ and a smooth segment $[p, q]$ which meet to each other transversally and exactly at $\{p, q\}$. Let $\pi_{1}: \mathfrak{R}^{2} \rightarrow \mathfrak{R}$ be given by $\pi_{1}(x, y)=x$. Suppose that $C$ is contained in the complement of the compact ball of radius $\rho+1$ centered at the origin. If $\nabla f$ points inward (resp. outward) the disc bounded by $C$, along $(p, q)_{f}$, then $\inf \left(\pi_{1}\left([p, q]_{f}\right)\right)=x_{2} \geq$ $x_{1}=\inf \left(\pi_{1}([p, q])\right)\left(\right.$ resp. $\sup \left(\pi_{1}\left([p, q]_{f}\right)\right)=\tilde{x}_{2} \leq \tilde{x}_{1}=\sup \left(\pi_{1}([p, q])\right)$. 
Consider only the case in which $\nabla f$ points inward $C$ along $(p, q)_{f}$. Assume by contradiction that $\inf \left(\pi_{1}\left([p, q]_{f}\right)\right)=x_{2}<x_{1}$ and take a point $\left(x_{2}, y_{2}\right) \in[p, q]_{f}$. See fig. 2.3. By the assumptions, there exists a vector field $Y: C \rightarrow \mathfrak{R}^{2}$ which points inward the disc bounded by $C$ and which coincides with $\nabla f$ in the complement of a small neighbourhood $V$ of $[p, q]$. We may take $V$ so small that $\left(x_{2}, y_{2}\right) \notin V$. As the half plane $\left\{(x, y) \in \mathfrak{R}^{2}: x<x_{2}\right\}$ is disjoint of $C$ and $\nabla f$ points inward $C$ at $\left(x_{2}, y_{2}\right)$ we must have that $\nabla f\left(x_{2}, y_{2}\right) \in(0, \infty) \times\{0\}$. However this is a contradiction with the $\rho$-obstruction property.

Let $S^{1}=\{z \in \mathbf{C}:|z|=1\}$, we shall denote by $\operatorname{cl}\left(\mathfrak{R}^{2}\right)$ the compact space made up of the (disjoint) union of $\mathfrak{R}^{2}$ and $S^{1}$ and constructed in the following way: Let $\varphi:[0,1) \rightarrow[0, \infty)$ be a smooth diffeomorphism which is the identity in a small neighbourhood of 0 . Let $G:\{z \in \mathbf{C}:|z|<1\} \rightarrow \mathfrak{R}^{2}$ be the diffeomorphism given by $G(x+i y)=\varphi\left(x^{2}+y^{2}\right) \cdot(x, y)$. By identifying $(x+i y)$ with $G(x+i y)$ and by borrowing from $\{z \in \mathbf{C}:|z| \leq 1\}$ its topology, we shall obtain the required topology for $\operatorname{cl}\left(\mathfrak{R}^{2}\right)$. Given a half trajectory $F_{p}^{\delta}$ of $\nabla f^{\perp}$, it follows from the Poincare-Bendixon Theorem and from the fact that $\nabla f^{\perp}$ has no singularities that the limit set $\mathcal{L}\left(F_{p}^{\delta}\right)$ of $F_{p}^{\delta}$, as a subset of $\operatorname{cl}\left(\mathfrak{R}^{2}\right)$, is either $S^{1}$ or a nonempty closed subinterval of it. Proper subintervals of $S^{1}$ will be denoted in the following way: given two real numbers $\theta_{1} \leq \theta_{2}$ such that $\theta_{2}-\theta_{1}<2 \pi$,

$$
\begin{aligned}
& {\left[e^{i \theta_{1}}, e^{i \theta_{2}}\right]=\left\{e^{i \theta}: \theta_{1} \leq \theta \leq \theta_{2}\right\}} \\
& \left(e^{i \theta_{1}}, e^{i \theta_{2}}\right]=\left\{e^{i \theta}: \theta_{1}<\theta \leq \theta_{2}\right\}
\end{aligned}
$$

etc.

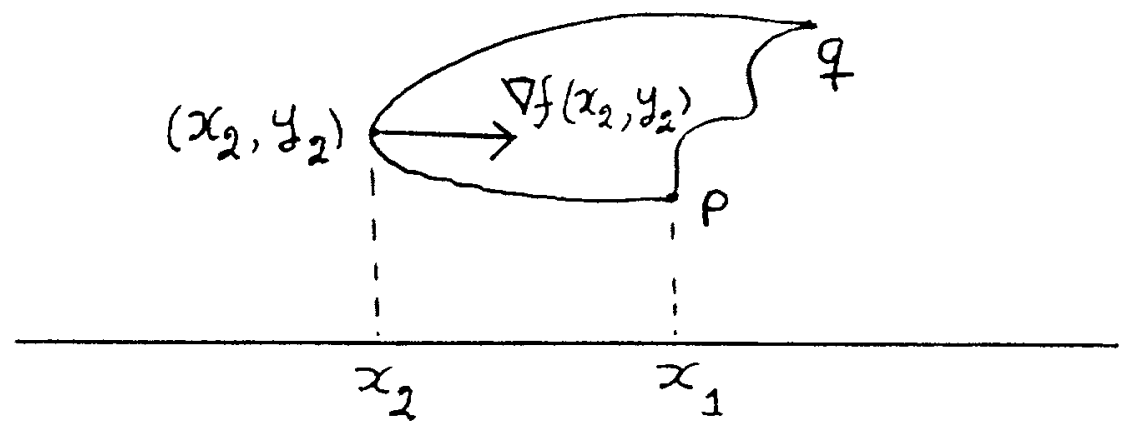

FIG. 2.3

LEMMA 2.4. - Let $\pi_{1}: \mathfrak{R}^{2} \rightarrow \mathfrak{R}$ be given by $\pi_{1}((x, y))=x$. Suppose that $F_{p_{1}}^{+}, F_{p_{3}}^{-}$and $\left[p_{1}, p_{3}\right]$ are the edges of a half-Reeb component $C$. Suppose 
that $C$ is contained in the complement of the compact ball of radius $\rho+1$ centered at the origin. If $\nabla f$ points inward $C$ (resp. outward $C$ ) along $F_{p_{1}}^{+} \cup F_{p_{3}}^{-}$, then

$$
C \subset\left\{(x, y) \in \mathfrak{R}^{2}: x \geq x_{1}=\inf \left(\pi_{1}\left(\left[p_{1}, p_{3}\right]\right)\right\}\right.
$$

(resp. $C \subset\left\{(x, y) \in \mathfrak{R}^{2}: \mathfrak{x} \leq \mathfrak{x}_{2}=\sup \left(\pi\left(\left[\mathfrak{p}_{1}, \mathfrak{p}_{3}\right]\right)\right\}\right)$. In particular

$$
\left[\theta_{1}^{-}, \theta_{1}^{+}\right] \cup\left[\theta_{3}^{-}, \theta_{3}^{+}\right] \subset[-\pi / 2, \pi / 2]
$$

(resp. $\left.\left[\theta_{1}^{-}, \theta_{1}^{+}\right] \cup\left[\theta_{3}^{-}, \theta_{3}^{+}\right] \subset[\pi / 2,3 \pi / 2]\right)$ where

$$
\mathcal{L}\left(F_{p_{1}}^{+}\right)=\left[e^{i \theta_{1}^{-}}, e^{i \theta_{1}^{+}}\right] \quad \text { and } \quad \mathcal{L}\left(F_{p_{3}}^{-}\right)=\left[e^{i \theta_{3}^{-}}, e^{i \theta_{3}^{+}}\right]
$$

Moreover,

$$
\theta_{1}^{+} \geq \theta_{3}^{+} \quad \text { and } \quad \theta_{1}^{-} \geq \theta_{3}^{-}
$$

(resp. $\theta_{1}^{+} \leq \theta_{3}^{+}$and $\theta_{1}^{-} \leq \theta_{3}^{-}$).

Proof. - Consider only the case in which $\nabla f$ points inward $C$ along $F_{p_{1}}^{+} \cup F_{p_{3}}^{-} \backslash\left\{p_{1}, p_{3}\right\}$. Let $\gamma:[1,3] \rightarrow\left[p_{1}, p_{3}\right]$ be a smooth parametrization such that $\gamma(s)=p_{s}$, for $s \in\{1,3\}$.

Let $\pi_{1}: \mathfrak{R}^{2} \rightarrow \mathfrak{R}$ be the projection $(x, y) \rightarrow x$. If $1<s<t<3$ are such that $\gamma(s)$ and $\gamma(t)$ are the endpoints of an arc of trajectory of $\nabla f^{\perp}$ that is contained in $C$, we may apply Lemma 2.3 to the simple closed curve $[\gamma(s), \gamma(t)]_{f} \cup \gamma([s, t])$ to conclude that $\inf \left(\pi_{1}\left([\gamma(s), \gamma(t)]_{f}\right)\right) \geq$ $\inf \left(\pi_{1}(\gamma([s, t]))\right)$. It follows from this that $(a)$ and $(b)$ of this lemma are true.

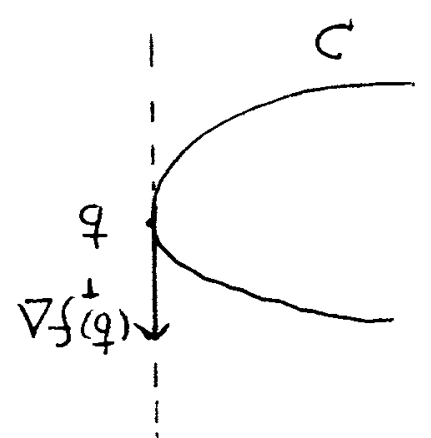

FIG. 2.4 


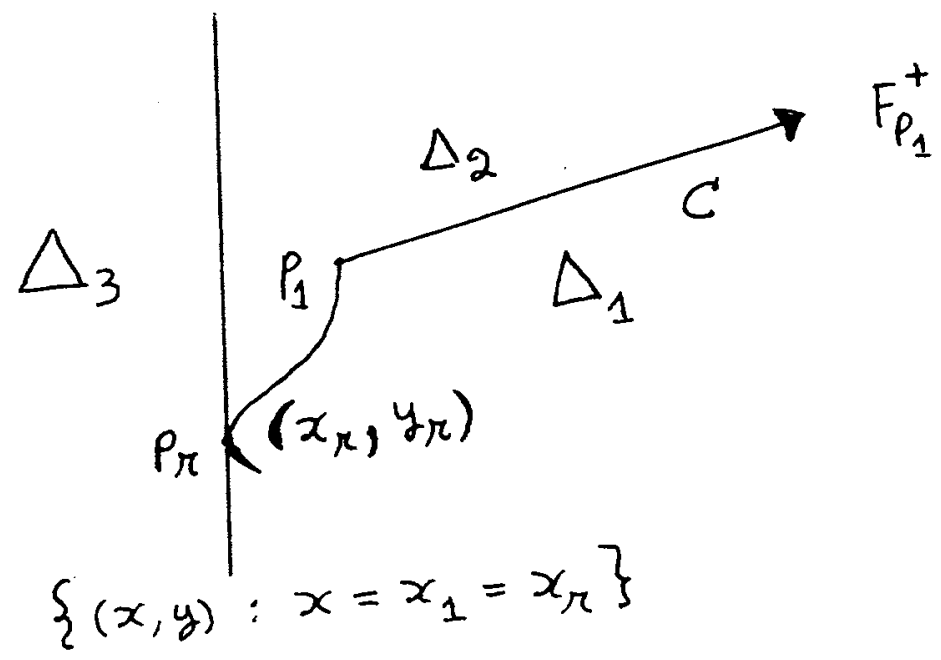

FIG. 2.5

(1) $F_{p_{1}}^{+}$may meet $\left\{(x, y): x=x_{1}\right\}$ at most at $p_{1}$.

Otherwise, if $q=\left(x_{1}, y_{1}\right) \in F_{p_{1}}^{+} \backslash\left\{p_{1}\right\}$, then, by $(a)$ and $(b)$ of this lemma, $\nabla f^{\perp}(q)$ would be tangent to $\left\{(x, y): x=x_{1}\right\}$. By the $\rho$-obstruction property, $\nabla f^{\perp}(q) \in\{0\} \times(-\infty, 0)$. As $C$ is on the right of $F_{p_{1}}^{+}$, there would be points of $C$ in the half plane $\left\{(x, y): x<x_{2}\right\}$. See fig. 2.4. This contradiction with $(a)$ and $(b)$ proves (1).

Let $\gamma(r)=p_{r}=\left(x_{r}, y_{r}\right)$. By what was said above, the union of the curves $\left\{x=x_{r}\right\}$ and $\left[p_{1}, p_{r}\right]_{\gamma} \cup F_{p_{1}}^{+}$separate their complement in $\mathfrak{R}^{2}$ into three open connected components $\Delta_{1}, \Delta_{2}, \Delta_{3}$ where

$$
\begin{aligned}
& \partial \Delta_{1}=\left\{x=x_{r}, y \leq y_{r}\right\} \cup\left[p_{1}, p_{r}\right]_{\gamma} \cup F_{p_{1}}^{+}, \\
& \partial \Delta_{2}=\left\{x=x_{r}, y \geq y_{r}\right\} \cup\left[p_{1}, p_{r}\right]_{\gamma} \cup F_{p_{1}}^{+}, \\
& \partial \Delta_{3}=\left\{x=x_{r}\right\} .
\end{aligned}
$$

As $C$ is on the right of $F_{p_{1}}^{+}, C \subset \Delta_{1} \cup \partial \Delta_{1}$. See fig. 2.5. Therefore, (c) is true.

LEMMA 2.5. - Let $\gamma:[0,4] \rightarrow \mathfrak{R}^{2}$ be a smooth curve such that $(f \circ \gamma)^{\prime}(s)$ vanishes exactly at $s=2$ and $(f \circ \gamma)^{\prime \prime}(2)=c \neq 0$. If $\gamma([1,3])$ is the compact edge of a half-Reeb component $C$ of $\nabla f^{\perp}$ then, for some $\varepsilon>0$, there are continuous monotone functions

$$
L, R, \tau: \quad(-\varepsilon, \varepsilon) \rightarrow[0,4]
$$

such that $L(0)=1, \tau(0)=2, R(0)=3$ and 
(a) $\frac{d}{d s}\left(f_{\theta} \circ H_{\theta} \circ \gamma(s)\right)$ vanishes exactly at $s=\tau(\theta)$ and, for all $\theta \in(-\varepsilon, \varepsilon)$, $\left|\frac{d^{2}}{d s^{2}}\left(f_{\theta} \circ H_{\theta} \circ \gamma(\tau(\theta))\right)-\frac{c}{2} \neq 0\right|$;

(b) $H_{\theta} \circ \gamma([L(\theta), R(\theta)])$ is the compact edge of a half-Reeb component $C(\theta)$ of $\nabla f^{\perp}$;

(c) The following possible properties do not depend on $\theta$ :

(c.1) $C(\theta)$ is on the left of $H_{\theta} \circ \gamma$

(c.2) $C(\theta)$ is on the right of $H_{\theta} \circ \gamma$

(c.3) $\nabla f_{\theta}$ points into $C(\theta)$ (along its non-compact edges)

(c.4) $\nabla f_{\theta}$ points out of $C(\theta)$ (along its non-compact edges);

(d) If $C(\theta)$ is on the left (resp. on the right) of $H_{\theta} \circ \gamma$ then $L(\theta), \tau(\theta), R(\theta)$ are strictly decreasing (resp. strictly increasing) functions;

(e) If $F_{r}^{+}$and $F_{\tilde{r}}^{-}$, with $r, \tilde{r} \in\left\{p_{1}, p_{3}\right\}$, are the positive and negative trajectories which are the edges of $C$, then $\left\{e^{i \frac{\pi}{2}}, e^{i \frac{3 \pi}{2}}\right\}$ is disjoint of $\mathcal{L}\left(F_{r}^{+}\right) \cup \mathcal{L}\left(F_{\tilde{r}}^{-}\right)$.

Proof. - As

we have that

$$
\left(\begin{array}{c}
f_{\theta} \\
g_{\theta}
\end{array}\right)=X_{\theta}=H_{\theta} \circ X \circ H_{\theta}^{-1}
$$

(1) $f_{\theta}\left(H_{\theta}(\gamma(s))\right)=\cos \theta \cdot(f \circ \gamma)(s)-\sin \theta \cdot(f \circ \gamma)(s)$, and $D X_{\theta}\left(H_{\theta}(\gamma(s))\right)=H_{\theta} \circ D X(\gamma(s)) \circ H_{\theta}^{-1}$.

Hence,

$$
\begin{aligned}
\frac{d}{d s}\left(\begin{array}{c}
f_{\theta}\left(H_{\theta}(\gamma(s))\right) \\
g_{\theta}\left(H_{\theta}(\gamma(s))\right)
\end{array}\right) & =\frac{d}{d s}\left(X_{\theta} \circ H_{\theta}(\gamma(s))\right) \\
& =D X_{\theta}\left(H_{\theta}(\gamma(s))\right) \cdot \frac{d}{d s}\left(H_{\theta} \circ \gamma(s)\right) \\
& =H_{\theta} \circ D X(\gamma(s)) \cdot \gamma^{\prime}(s) \\
& =H_{\theta}\left(\frac{d}{d s}(X \circ \gamma(s))\right) \\
& =H_{\theta}\left(\begin{array}{c}
(f \circ \gamma)^{\prime}(s) \\
(g \circ \gamma)^{\prime}(s)
\end{array}\right) \\
& =\left(\begin{array}{c}
\cos \theta \cdot(f \circ \gamma)^{\prime}(s)-\sin \theta \cdot(g \circ \gamma)^{\prime}(s) \\
\sin \theta \cdot(f \circ \gamma)^{\prime}(s)+\cos \theta \cdot(g \circ \gamma)^{\prime}(s)
\end{array}\right)
\end{aligned}
$$

Thus,

$$
\begin{aligned}
\Delta(\theta, s) & =\frac{d}{d s}\left(f_{\theta}\left(H_{\theta}(\gamma(s))\right)\right. \\
& =\cos \theta \cdot(f \circ \gamma)^{\prime}(s)-\sin \theta \cdot(g \circ \gamma)^{\prime}(s)
\end{aligned}
$$


As $\Delta(0,2)=0$ and $\frac{\partial}{\partial s} \Delta(0,2)=(f \circ \gamma)^{\prime \prime}(2)$, it follows, from the Implicit Function Theorem, that there exists a $C^{1}$ function

$$
\tau: \quad(-\varepsilon, \varepsilon) \rightarrow[0,4]
$$

such that $\Delta(\theta, \tau(\theta)) \equiv 0$. It follows that

$$
\frac{\partial \Delta}{\partial \theta}(0,2)+\frac{\partial \Delta}{\partial s}(0,2) \cdot \tau^{\prime}(0)=-(g \circ \gamma)^{\prime}(2)+(f \circ \gamma)^{\prime \prime}(2) \cdot \tau^{\prime}(0)=0
$$

and so,

$$
\tau^{\prime}(0)=\frac{(g \circ \gamma)^{\prime}(2)}{(f \circ \gamma)^{\prime \prime}(2)}
$$

Now we claim that

(4) If $C$ is on the left of $\gamma$, then $\tau^{\prime}(0)<0$.

In fact, suppose first that $\nabla f$ points inward $C$. The assumptions imply that $(f \circ \gamma)$ has a maximum at $s=2$ and so $(f \circ \gamma)^{\prime \prime}(2)<0$. Also as both $\left\{\nabla f(\gamma(2)), \gamma^{\prime}(2)\right\}$ and $\{\nabla f(\gamma(2)), \nabla g(\gamma(2))\}$ are positive basis, it must be that $(g \circ \gamma)^{\prime}(2)>0$. Hence $\tau^{\prime}(0)<0$.

Suppose this time that $\nabla f$ points outward $C$. Similarly as above we may see that $(f \circ \gamma)^{\prime \prime}(2)>0$ and $(g \circ \gamma)^{\prime}(2)<0$ and so $\tau^{\prime}(0)<0$. This proves (4). See fig. 2.6.

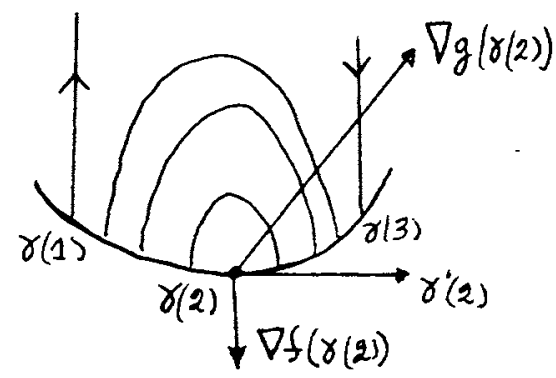

(a)

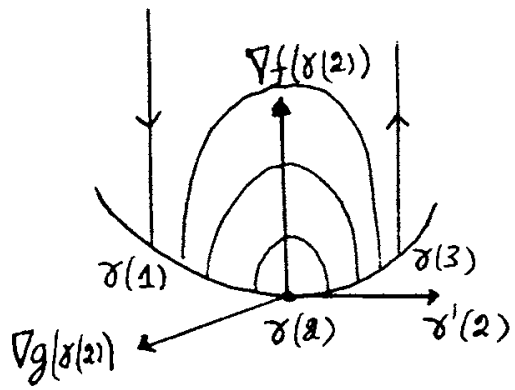

(b)

FIG. 2.6

Proceeding in this way, we may obtain that $(a)$ is true and that if $C$ in on the left (resp. on the right) of $\gamma$ then $\tau=\tau(\theta)$ is a strictly decreasing (resp. increasing) function of $\theta$. 
To prove $(b)$, consider only the case in which (5) $C$ in on the left of $\gamma$ and $\nabla f$ points inward $C$.

Let $0<\delta<1$ be given. By the assumptions of the lemma, $f \circ \gamma(1-\delta) \notin f \circ \gamma([1,3])$. Therefore, there exists $\varepsilon>0$ such that (6) For all $\theta \in[-\varepsilon, \varepsilon], f_{\theta}\left(H_{\theta} \circ \gamma(1-\delta)\right) \notin f_{\theta}\left(H_{\theta} \circ \gamma([1,3])\right)$.

As $\nabla f_{\theta}^{\perp}$ depends differentiably on $\theta$ and by the behaviour of the trajectories of $\nabla f$ in $C$, we may see that if $\varepsilon>0$ is small enough, for each $\theta \in(-\varepsilon, \varepsilon)$, there exists an orientation reversing continuous map

$$
T_{\theta}: \quad(L(\theta), \tau(\theta)] \rightarrow[\tau(\theta), 4]
$$

such that

(7) $T_{\theta}(\tau(\theta))=\tau(\theta)$ and, for all $s \in(L(\theta), \tau(\theta)]$, there is an arc of trajectory $M_{s}^{\theta}$ of $\nabla f_{\theta}^{\perp}$ starting at $H_{\theta}(\gamma(s))$ and ending at $H_{\theta}\left(\gamma\left(T_{\theta}(s)\right)\right)$.

As $f_{\theta}$ is constant along each arc $M_{s}^{\theta}$ of $\nabla f_{\theta}^{\perp}$, it follows from (6) that $1-\delta \leq L(\theta)$. It follows from Lemma 2.2 that if $\theta \in(0, \varepsilon)$, we may take $L(\theta)<1$ and so $R(\theta)=\lim _{s \rightarrow L(\theta)} T_{\theta}(s)<3$. Therefore if $L(\theta)$ is determined by the fact that $(L(\theta), \tau(\theta)]$ is the maximal interval where $T_{\theta}$ can be defined so to satisfy $(b)$, we will have that:

(8) if $\theta \in(0, \varepsilon), 1-\delta \leq L(\theta)<1$.

Similarly, using Lemma 2.2 , we may prove that

(9) If $\varepsilon>0$ is small enough and $\theta \in(-\varepsilon, 0)$, then $1<L(\theta)<1+\delta$.

This proves that $\theta \rightarrow L(\theta)$ is continuous and strictly decreasing. Similarly, we may conclude that

(10) $R(\theta)$ is continuous and strictly decreasing.

Under these circumstances, $(b),(c)$ and $(d)$ are immediate.

Let $B(0 ; \rho+1)$ be the compact ball of radius $\rho+1$ centered at the origin. To prove $(e)$, observe first that $C$ contains a half-Reeb component $\tilde{C} \subset \mathfrak{R}^{2} \backslash B(0 ; \rho+1)$ such that the edges of $\tilde{C}$, which are half-trajectories, are contained in $F_{r}^{+} \cup F_{\tilde{r}}^{-}$. As the conclusions in $(e)$ refer only to the assymptotic limit sets $\mathcal{L}\left(F_{r}^{+}\right)$and $\mathcal{L}\left(F_{\tilde{r}}^{-}\right)$, we may suppose in the remainder of the proof that $C$ itself is disjoint of $B(0 ; \rho+1)$.

To proceed with the proof of $(e)$, consider only the case in which $\nabla f$ points inward $C$ along $\partial_{1} C \backslash\left[p_{1}, p_{3}\right]_{\gamma}$ and $C$ is on the left of $\gamma$. This implies that

(11) $p_{r}=p_{1}$.

As the required conclusion in $(e)$ is related to $F_{p_{1}}^{+}$and $F_{p_{3}}^{-}$, it can be assumed, by means of a small perturbation of $\gamma$, that when $\varepsilon>0$ is small and $\theta \in[0, \varepsilon)$,

(12) Both $\nabla f_{\theta}^{\perp}$ and the vertical foliation (i.e. the one made up of the lines $\{(x, y): x=$ constant $\})$ meet transversally $H_{\theta} \circ \gamma$ at the 
points $H_{\theta} \circ \gamma(0)$ and $H_{\theta} \circ \gamma(4)$. Moreover, there is exactly one point $m_{\theta}=\left(x_{\theta}, y_{\theta}\right) \in H_{\theta} \circ \gamma([0,4])$, depending differentiably on $\theta$, such that $x_{\theta}=\inf \left(\pi_{1}\left(H_{\theta} \circ \gamma([0,4])\right)\right)$ and if $m_{\theta} \notin\left\{H_{\theta} \circ \gamma(0), H_{\theta} \circ \gamma(4)\right\}$ then $H_{\theta} \circ \gamma$ has quadratic contact with $\left\{(x, y): x=x_{\theta}\right\}$ at $\left(x_{\theta}, y_{\theta}\right)$.

Now,

(13) Fix $\theta \in(0, \varepsilon)$.

Assume, by contradiction, that (14) $e^{i \pi / 2} \in \mathcal{L}\left(F_{p_{1}}^{+}\right)$.

We claim that if $\varepsilon>0$ is small enough.

(15) $H_{\theta}\left(F_{p_{1}}^{+}\right)$crosses $\left\{(x, y): x=x_{\theta}\right\}$ at a point $n_{\theta}=\left(x_{\theta}, z_{\theta}\right)$ with $z_{\theta}>y_{\theta}$. See fig. 2.7 .

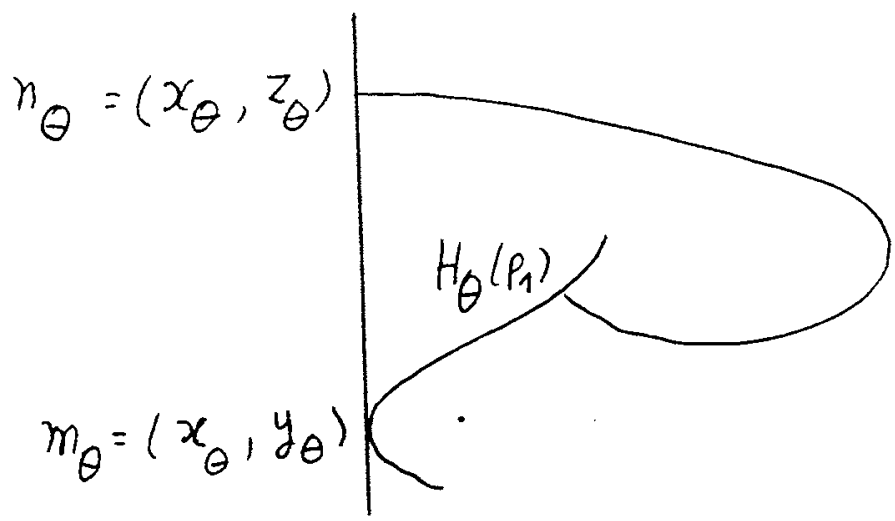

FIG. 2.7

In fact, first observe that if $p_{1}=m_{0}=\left(x_{0}, y_{0}\right)$, then, by the $\rho$-obstruction property, $\nabla f^{\perp}\left(p_{1}\right)$ has positive first coordinate and therefore, if $\varepsilon>0$ is small and so $\left(x_{\theta}, y_{\theta}\right)$ is very close to $H_{\theta}\left(x_{0}, y_{0}\right), H_{\theta}\left(F_{p_{1}}^{+} \backslash\left\{p_{1}\right\}\right)$ cannot meet the subsegment of $H_{\theta} \circ \gamma$ with endpoints $H_{\theta}\left(x_{0}, y_{0}\right)$ and $\left(x_{\theta}, y_{\theta}\right)=m_{\theta}$. If $p_{1} \neq m_{0}$, then $\varepsilon>0$ can be taken so small that $\left(x_{\theta}, y_{\theta}\right),\left(x_{0}, y_{0}\right)$ and $H_{\theta}\left(\left(x_{0}, y_{0}\right)\right)$ are so close to each other that $H_{\theta}\left(F_{p_{1}}^{+}\right)$cannot meet the subsegment of $H_{\theta} \circ \gamma$ with endpoints $H_{\theta}\left(x_{0}, y_{0}\right)$ and $m_{\theta}$. (See fig. 2.8). As by Lemma 2.4 and (12), $H_{\theta}\left(F_{p_{1}}^{+}\right) \subset H_{\theta}\left(\left\{(x, y): x \geq x_{0}\right\}\right)$ we must have that $H_{\theta}\left(F_{p_{1}}^{+}\right)$does not meet $\left\{(x, y): x=x_{\theta}\right.$ and $\left.y \leq y_{\theta}\right\}$. Therefore, as by (14),

$$
e^{i\left(\frac{\pi}{2}+\theta\right)} \in \mathcal{L}\left(H_{\theta}\left(F_{p_{1}}^{+}\right)\right),
$$

it must be that (15) is true.

Let consider the disc $D$ bounded by the straight line segment $\left[m_{\theta}, n_{\theta}\right]$, the subarc $\left[H_{\theta}\left(p_{1}\right), n_{\theta}\right]$ of $H_{\theta}\left(F_{p_{1}}^{+}\right)$and the subsegment $\left[H_{\theta}\left(p_{1}\right), m_{\theta}\right]_{H_{\theta} \circ \gamma}$ Vol. $12, n^{\circ} 6-1995$ 
of $H_{\theta} \circ \gamma$. If $\left[H_{\theta}\left(p_{1}\right), n_{\theta}\right]$ is provided with the orientation induced by that of $H_{\theta}\left(F_{p_{1}}^{+}\right)$, then as $\left[m_{\theta}, n_{\theta}\right]$ is on the left,

(16) $D$ is on the left of $\left[H_{\theta}\left(p_{1}\right), n_{\theta}\right]$.

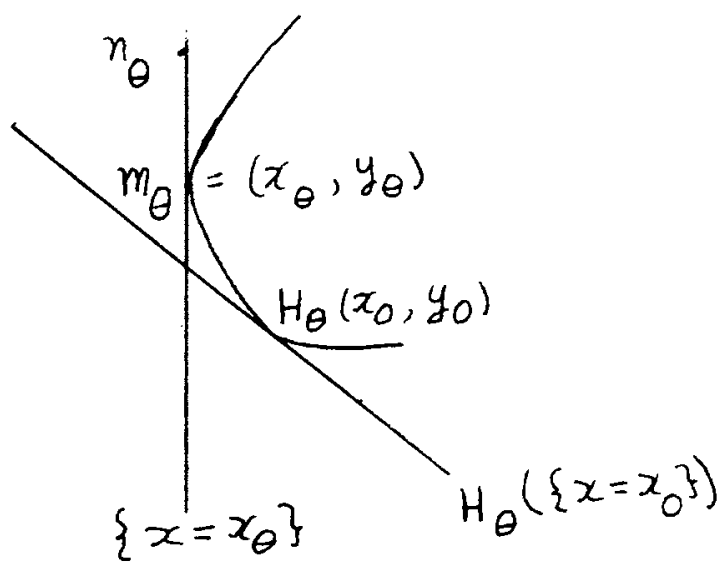

FIG. 2.8

It follows from the Lemma 2.2 that the oriented arcs $M_{s}^{\theta}$, introduced in (7), meet $\left[H_{\theta}\left(p_{1}\right), n_{\theta}\right]$ exactly once and transversally and, when they do this, it is from the left of $\left[H_{\theta}\left(p_{1}\right), n_{\theta}\right]$. Let $K_{s}^{\theta}$ be the subarc of $M_{s}^{\theta}$ starting at $H_{\theta}(\gamma(s))$ and ending at $M_{s}^{\theta} \cap\left[H_{\theta}\left(p_{1}\right), n_{\theta}\right]$. Under these circumstances, it follows from (16) that, for $s<1$ close to $1, K_{s}^{\theta} \subset D$. We claim that (17) $s^{*}:=\inf \left\{s: K_{s}^{\theta} \subset D\right\}=L(\theta)$

Assume, by contradiction, that $L(\theta)<s^{*}$. Then $K_{s^{*}}^{\theta}$ is disjoint of $\left[m_{\theta}, n_{\theta}\right]$, because otherwise any arc $K_{s}^{\theta}$, with $s<s^{*}$ close to $s^{*}$, would meet $\left\{(x, y): x<x_{\theta}\right\}$ contradicting Lemma 2.3. Also $K_{s^{*}}^{\theta}$ is disjoint of $\left[H_{\theta}\left(p_{1}\right), m_{\theta}\right]_{H_{\theta} \circ \gamma}$, because otherwise $K_{s^{*}}^{\theta} \cap\left[H_{\theta}\left(p_{1}\right), m_{\theta}\right]=\gamma(\tau(\theta))$. However, by $(a), f_{\theta}(\gamma(\tau(\theta))) \neq f_{\theta}\left(K_{s^{*}}^{\theta}\right)$. This implies (17).

It follows from the trajectory structure of $\left.\nabla f^{\perp}\right|_{C}$ and Lemma 2.2 that the arc of trajectory of $\nabla f_{\theta}^{\perp}$ starting at a point of $\left[H_{\theta}\left(p_{1}\right), n(\theta)\right]$ must reach $H_{\theta} \circ \gamma$. This and (17) imply that there is an $\operatorname{arc} M_{L(\theta)}^{\theta}$ of $\nabla f_{\theta}^{\perp}$ which contradicts the assumed properties of $L(\theta)$. Therefore $(e)$ is proved.

LEMMA 2.6. - Let $\gamma:[0,3] \rightarrow \mathfrak{R}^{2}$ be a smooth curve such that $\gamma$ restricted to $[0,1]$ is transversal to $\nabla f^{\perp}$ and $\gamma([1,3])$ is the compact edge of the half-Reeb component $C$.

(a) If $C$ is on the left (resp. on the right) of $\gamma$ and its noncompact edges are $F_{\gamma(1)}^{+}$and $F_{\gamma(3)}^{-}$then

$$
\mathcal{L}\left(F_{\gamma(0)}^{+}\right) \subset\left(e^{-i \pi / 2}, e^{i \pi / 2}\right]
$$


(resp. $\mathcal{L}\left(F_{\gamma(0)}^{+}\right) \subset\left[e^{i \pi / 2}, e^{3 i \pi / 2}\right)$.

(b) If $C$ is on the left (resp. on the right) of $\gamma$ and its noncompact edges are $F_{\gamma(1)}^{-}$and $F_{\gamma(3)}^{+}$then

$$
\mathcal{L}\left(F_{\gamma(0)}^{-}\right) \subset\left(e^{i \pi / 2}, e^{3 i \pi / 2}\right]
$$

(resp. $\left.\mathcal{L}\left(F_{\gamma(0)}^{-}\right) \subset\left[e^{-i \pi / 2}, e^{i \pi / 2}\right)\right)$

See fig. 2.9 .
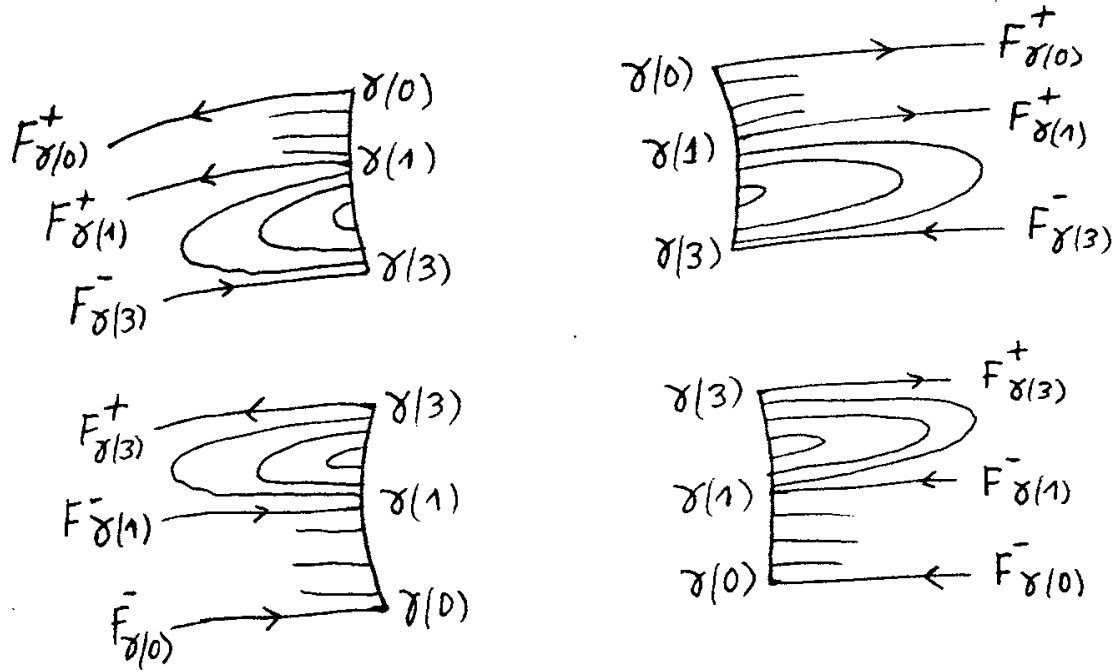

FIG. 2.9

Proof. - Consider only the case in which $F_{\gamma(1)}^{+}$is an edge of $C$ and that $C$ in on the left on $\gamma$.

In the following, $B(0 ; \tilde{\rho})$ will denote the compact ball of radius $\tilde{\rho}$ centered at the origin. As the conclusions in $(e)$ refer only to the assymptotic limit sets $\mathcal{L}\left(F_{r}^{+}\right), \mathcal{L}\left(F_{\tilde{r}}^{-}\right)$and $\mathcal{L}\left(F_{\gamma(0)}^{+}\right)$, by the same arguments used in the proof of Lemma $2.5(e)$, we may suppose that $C \cup F_{\gamma(0)}^{+}$is disjoint of $B(0 ; \rho+3)$. Notice that $\gamma([0,1])$ may or may not meet $B(0 ; \rho)$.

Let $\left(x_{0}, y_{0}\right)$ be the point of $\gamma([0,3])$ such that $x_{0}=\inf \left(\pi_{1}(\gamma([0,3]))\right)$ and $\left\{\left(x_{0}, y\right): y>y_{0}\right\}$ is disjoint of $\gamma([0,3])$. Here $\pi_{1}: \mathfrak{R}^{2} \rightarrow \mathfrak{R}$ is the projection on the first coordinate.

Suppose by contradiction that

(1) $\mathcal{L}\left(F_{\gamma(0)}^{+}\right)$is not contained in $\left(e^{-i \pi / 2}, e^{i \pi / 2}\right]$. 
By the same argument as that of the proof of (c) of Lemma 2.4, $F_{\gamma(0)}^{+}$ must cross transversally $\left\{x=x_{0}\right\}$ at some point $\left(x_{0}, z_{0}\right)$ with $z_{0}>y_{0}$. This implies that there exists $p \in F_{\gamma(0)}^{+}$close to $\left(x_{0}, z_{0}\right)$ such that (2) $x_{0}=\inf \left(\pi_{1}(\gamma([0,3]))\right)>\pi_{1}(p), \nabla f^{\perp}(p)$ has negative first coordinate and for some $\varepsilon>0$ and for all $z \in\left[\pi_{1}(p)-\varepsilon, \pi_{1}(p)+\varepsilon\right]$, the arc of trajectory $[\gamma(0), p]_{f}$ of $\nabla f^{\perp}$ meets $\{(x, y): x=z\}$ exactly once and transversally at a point belonging to $\mathfrak{R} \backslash B(0 ; \rho+2)$. See fig. 2.10 .

As, by Lemmas $2.3,2.4$ and $2.5(e), \mathcal{L}\left(F_{\gamma(1)}^{+}\right) \subset\left(e^{-i \pi / 2}, e^{i \pi / 2}\right)$ and $F_{\gamma(1)}^{+} \subset\left\{(x, y): x \geq x_{0}\right\}$, using an argument similar to that of (2) above, there exists $q \in F_{\gamma(1)}^{+}$such that

(3) $\sup \left(\pi_{1}(\gamma([0,3]))\right)<\pi_{1}(q), \nabla f^{\perp}(q)$ has positive first coordinate and for some $\varepsilon>0$ and for all $z \in\left[\pi_{1}(q)-\varepsilon, \pi_{1}(q)+\varepsilon\right]$, the arc of trajectory $[\gamma(0), p]_{f}$ of $\nabla f^{\perp}$ meets $\{(x, y): x=z\}$ exactly once and transversally at a point belonging to $\mathfrak{R} \backslash B(0 ; \rho+2)$. See fig. 2.10 .

The piecewise differentiable curve $[\gamma(0), p]_{f} \cup[\gamma(0), \gamma(1)]_{\gamma} \cup[\gamma(1), q]_{f}$ can be approximated by a smooth embedding $\lambda:[0,3] \rightarrow\left[\pi_{1}(p), \pi_{1}(q)\right] \times$ $\Re$, transversal to $\nabla f^{\perp}$ connecting $p=\lambda(0)$ with $q=\lambda(3)$, and in such a way that $\nabla f^{\perp}$ points to the left of $\lambda$ (i.e., for all $s \in[0,3],\left\{\lambda^{\prime}(s), \nabla f(\lambda(s)\}\right.$ is a negative basis) and for all $z \in\left[\pi_{1}(p), \pi_{1}(p)+\varepsilon\right] \cup\left[\pi_{1}(q)-\varepsilon, \pi_{1}(q)\right]$ (with $\varepsilon>0$ as in (2) and (3) above), $\lambda$ meets $\{(x, y): x=z\}$ exactly once and transversally. The curve $\lambda$ is represented in figure 2.10 by a dotted line.

The curve $\nabla f^{\perp} \circ \lambda:[0,3] \rightarrow \mathfrak{R}^{2} \backslash\{0\}$ connects the point $\nabla f^{\perp}(p)$ (having negative first coordinate) with the point $\nabla f^{\perp}(q)$ (having positive first coordinate).

It is not difficult to see that there is a smooth diffeotopy $t \rightarrow G_{t}$, $t \in[0,1]$, of $\mathfrak{R}^{2}$, such that

(4) $G_{0}$ is the identity map. For all $t \in[0,1], G_{t}$ restricted to the complement of $\left(\pi_{1}(p)+\varepsilon, \pi_{1}(q)-\varepsilon\right) \times \mathfrak{R}$ in $\mathfrak{R}^{2}$ is the identity map. The image of $G_{1} \circ \lambda$ is the graph of a function $\left[\pi_{1}(p), \pi_{1}(q)\right] \rightarrow \mathfrak{R}$.

This implies that if $Y(t)=\left(D G_{1}\right)_{\lambda(t)} \cdot \nabla f^{\perp}(\lambda(t))$, then $Y(t)$ points to the left of $G_{1} \circ \lambda$ and, by (4), it does not meet the ray $\{(0, y): y<0\}$.

There exists a neighborhood $V(p, q)$ of $\{p, q\}$ such that $\lambda$ is homotopic, with $V(p, q)$ held fixed, to a smooth embedding

$$
\mu: \quad[0,3] \rightarrow\left(\left[\pi_{1}(p), \pi_{1}(q)\right] \times \Re\right) \backslash B(0 ; \rho+1),
$$

in such a way that, for all $z \in\left[\pi_{1}(p), \pi_{1}(p)+\varepsilon\right] \cup\left[\pi_{1}(q)-\varepsilon, \pi_{1}(q)\right]$ (with $\varepsilon>0$ as in (2) and (3) above), $\mu$ meets $\{(x, y): x=z\}$ exactly once and transversally. See the curve $\mu$ in figure 2.10 .

The curve $\nabla f^{\perp} \circ \mu:[0,3] \rightarrow \mathfrak{R}^{2} \backslash\{0\}$ connects the point $\nabla f^{\perp}(p)$ (having negative first coordinate) with the point $\nabla f^{\perp}(q)$ (having positive 


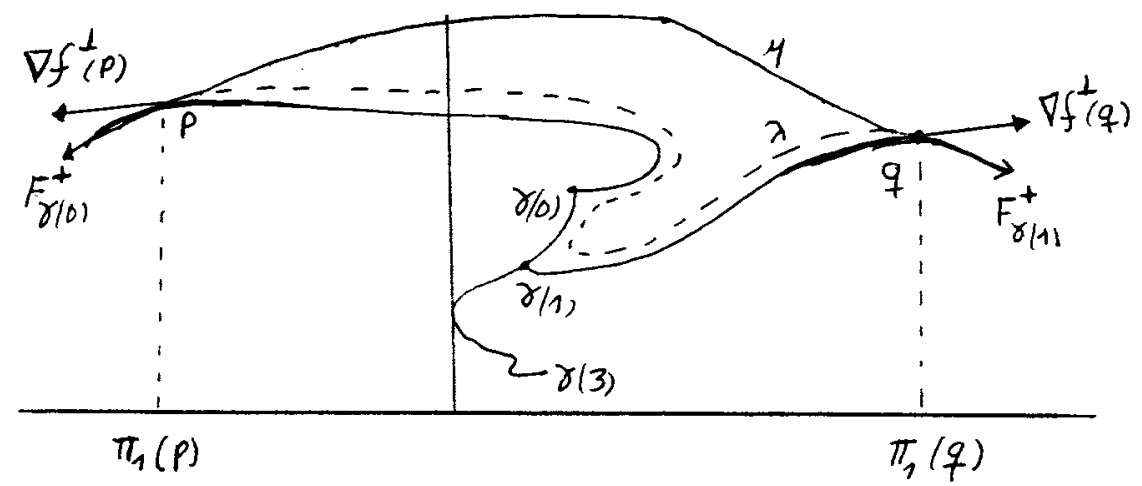

FIG. 2.10

first coordinate), and, by the $\rho$-obstruction property, the image of $\nabla f^{\perp} \circ \mu$ does not meet the ray $\{(0, y): y>0\}$. However this is a contradiction because the paths $Y$ and $\nabla f^{\perp} \circ \mu$ cannot be homotopic in $\mathfrak{R}^{2} \backslash\{0\}$.

Proof of Proposition 2.1. - If we assume that this proposition is false, we shall immediately reach a contradiction with lemmas $2.4,2.5(e)$ and 2.6.

\section{A CANONICAL DECOMPOSITION}

Let $a, p, q \in \mathfrak{R}^{2}$ be such that $F_{a}, F_{p}$ and $F_{q}$ are three different trajectories of $\nabla f^{\perp}$. We say that $F_{a}$ is between $F_{p}$ and $F_{q}$ if the last two are contained in different connected components of $\mathfrak{R}^{2} \backslash \mathfrak{F}_{\mathfrak{a}}$. Let $\left[F_{p}, F_{q}\right]$ be the closure of the connected component of $\mathfrak{R}^{2} \backslash\left(\mathfrak{F}_{\mathfrak{p}} \cup \mathfrak{F}_{\mathfrak{q}}\right)$ whose boundary is $F_{p} \cup F_{q}$. A transversal region of $\left[F_{p}, F_{q}\right]$ will be a connected component of the set formed by the union of $F_{p} \cup F_{q}$ with all the trajectories that are between $F_{p}$ and $F_{q}$.

Observe that the function $g$ is strictly monotone along any given trajectory $F_{p}$ of $\nabla f^{\perp}$. This follows immediately from the fact that if $v$ is a vector tangent to $F_{p}$ at some point $a$ then, as $\nabla f(a) \cdot v=0$ and $\operatorname{det}(D X(a))>0$, $\nabla g(a) \cdot v \neq 0$. In particular if $p$ and $q$ are singularities of $X$ then $F_{p}$ is disjoint of $F_{q}$.

PROPOSITION 3.1. - Let $p, q \in \mathfrak{R}^{2}$ be such that $X(p)=X(q)=0$. Among all smooth curves connecting $p$ with $q$, the curve $\gamma:[0,1] \rightarrow\left[F_{p}, F_{q}\right]$ with $\gamma(0)=p$ and $\gamma(1)=q$ has the least possible number of tangency points with $\nabla f^{\perp}$ if and only if there are numbers

$$
r_{0}=0 \leq l_{1}<r_{1} \leq l_{2}<r_{2} \leq \cdots \leq l_{n}<r_{n} \leq 1=l_{n+1},
$$


with $n \geq 1$, satisfying (a) and (b) below:

(a) For all $i \in\{1,2, \cdots, n\}, \gamma\left(\left[l_{i}, r_{i}\right]\right)$ is the compact edge of a half-Reeb component $C_{i}$.

(b) Given $i \in\{0,1, \cdots, n\}$, the union $T_{i}$ of all the trajectories $F_{\gamma(s)}$, with $s \in\left[r_{i}, l_{i+1}\right]$, is a transversal region of $\left[F_{p}, F_{q}\right]$. Also, for all $s \in\left[r_{i}, l_{i+1}\right]$,

$$
F_{\gamma(s)} \cap \gamma([0,1])=\{\gamma(s)\}
$$

\section{Furthermore,}

(c) Let $\gamma, \gamma^{*}:[0,1] \rightarrow\left[F_{p}, F_{q}\right]$ be smooth curves, connecting $\gamma(0)=$ $\gamma^{*}(0)=p$ with $\gamma(1)=\gamma^{*}(1)=q$ and having the least possible number of tangency points with $\nabla f^{\perp}$. Let $C_{i}$ and $T_{i}$ (resp. $C_{i}^{*}$ and $T_{i}^{*}$ ) be the half-Reeb components and transversal regions, respectively, associated to $\left(\left[F_{p}, F_{q}\right], \gamma\right)$ (resp. to $\left(\left[F_{p}, F_{q}\right], \gamma^{*}\right)$ ) and indexed orderly as in $(a)$ and $(b)$ above. Then $T_{i}=T_{i}^{*}$ and the intersection of $C_{i}$ and $C_{i}^{*}$ contains a half-Reeb component whose non-compact edges are contained in the non-compact edges of both $C_{i}$ and $C_{i}^{*}$.

The proof of Proposition 3.1 follows immediately from Lemmas 3.2-3.4.

LEMMA 3.2. - There exists a smooth curve $\gamma:[0,1] \rightarrow\left[F_{p}, F_{q}\right]$ with $\gamma(0)=p, \gamma(1)=q$ and there are real numbers

$$
0<t_{1}<t_{2}<\cdots<t_{n}<1
$$

such that:

(a) $(f \circ \gamma) r(s))=0$ if and only if $s \in\left\{t_{1}, t_{2}, \cdots, t_{n}\right\}$. Moreover, for all $s \in\left\{t_{1}, t_{2}, \cdots, t_{n}\right\},(f \circ \gamma) \prime \prime(s) \neq 0$; and

(b) A trajectory of $\nabla f^{\perp}$ can meet $\gamma$ at most at two points and, if so, it does it transversally.

Proof. - Let $\gamma:[0,1] \rightarrow\left[F_{p}, F_{q}\right]$ be a smooth curve connecting $\gamma(0)=p$ with $\gamma(1)=q$. By a small perturbation (if necessary) we may assume that $\gamma$ satisfies (a). As trajectories of $\nabla f^{\perp}$ can accumulate only at infinity, by a small perturbation of $\gamma$, we may assume that

(1) A trajectory may meet tangentially $\gamma$ at most at one point.

We will proceed to reconstruct $\gamma$ obtaining a new curve, still denoted by $\gamma$, but with less number of points of tangency with $\nabla f^{\perp}$ than the starting curve. To that end, consider first the following situation:

(2) There is a trajectory $F_{a}$ of $\nabla f^{\perp}$ that meets $\gamma$ transversally at $a$ and tangentially at some point $b$, and $\gamma \cap[a, b]_{f}=[a, b]_{\gamma} \cap[a, b]_{f}=\{a, b\}$.

In this situation we choose a small flow box $B$ centered at $[a, b]_{f}$, take two points $\tilde{a}, \tilde{b} \in B \cap \gamma$ such that $(\tilde{a}, \tilde{b})_{\gamma}$ contains properly $[a, b]$, and replace 
the original $[a, b]_{\gamma}$ by a new segment (drawn as a dotted line in Figure 3.1) contained in $B$ and transversal to $\nabla f^{\perp}$. Observe that $\nabla f^{\perp}$ had at least two tangency points with the previous $[a, b]_{\gamma}$ : one at $b$ and the other at some point $c \in(a, b)_{\gamma}$. As $\left[F_{p}, F_{q}\right]$ is simply connected, the new curve constructed in this way is contained in $\left[F_{p}, F_{q}\right]$.

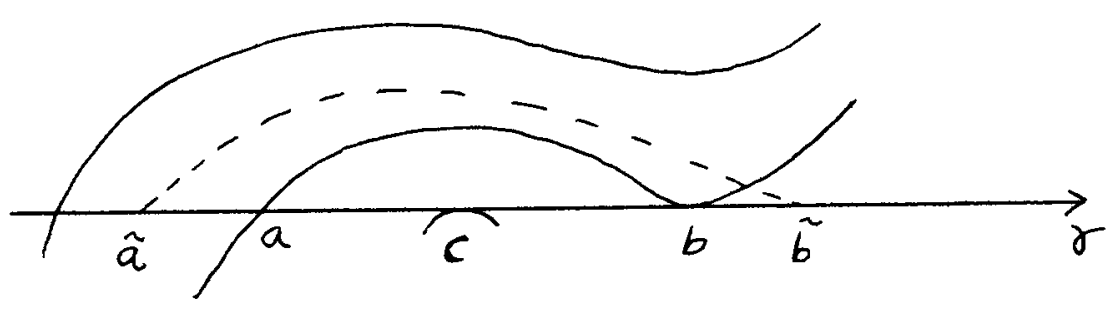

FIG. 3.1

By repeating when necessary the procedure above, we may assume that (3) Any trajectory of $\nabla f^{\perp}$ that meets $\gamma$ more than once must do it transversally everywhere.

Under condition (3), we shall decrease the number of tangency points using one of the procedures indicated at the items (4)-(6) below.

(4) There are three consecutive points $a_{1}, a_{2}$ and $a_{3}$ of a positive half-trajectory of $\nabla f^{\perp}$ such that $\gamma \cap\left[a_{1}, a_{3}\right]_{f}=\left[a_{1}, a_{3}\right]_{\gamma} \cap\left[a_{1}, a_{3}\right]_{f}=$ $\left\{a_{1}, a_{2}, a_{3}\right\}$.

In this situation we consider a small flow box $B$ centered at $\left[a_{1}, a_{3}\right]_{f}$, take a point $a_{4} \in B \cap \gamma$ such that $\left[a_{1}, a_{4}\right]_{\gamma}$ contains properly $\left[a_{1}, a_{3}\right]_{\gamma}$ and consider a segment transversal to $\nabla f^{\perp}$ contained in $B$ and joining $a_{1}$ with $a_{4}$. See fig. 3.2. Now, we put this new segment instead of the original $\left[a_{1}, a_{4}\right]_{\gamma}$. The resulting new segment (drawn with a dotted curve in figure 3.2) has at least two less tangency points of tangency with $\nabla f^{\perp}$ than the original $\gamma$ (which had a point of tangency at $\left(a_{1}, a_{2}\right)_{\gamma}$ and other at $\left.\left(a_{2}, a_{3}\right)\right)$.

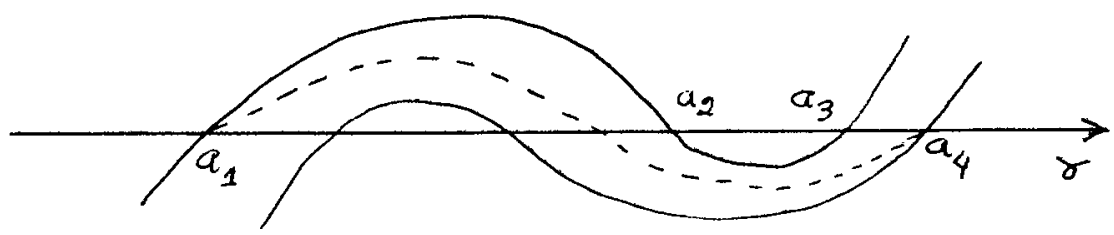

FiG. 3.2

Vol. 12, $n^{\circ}$ 6-1995. 
(5) There are three consecutive points $a_{1}, a_{2}$ and $a_{3}$ of a positive half-trajectory of $\nabla f^{\perp}$ such that $\gamma \cap\left[a_{1}, a_{3}\right]_{f}=\left[a_{1}, a_{2}\right]_{\gamma} \cap\left[a_{1}, a_{3}\right]_{f}=$ $\left\{a_{1}, a_{2}, a_{3}\right\}$.

In this situation, observe first that as $F_{a_{3}}^{+}$is entering through $a_{3}$ to the disc bounded by $\left[a_{1}, a_{3}\right]_{f} \cup\left[a_{1}, a_{3}\right]_{\gamma}$ it must leave this disc at a point $a_{4} \in\left(a_{1}, a_{3}\right)_{\gamma}$. It is easy to see that $\nabla f^{\perp}$ must meet tangentially somewhere each of the segments $\left(a_{1}, a_{4}\right)_{\gamma},\left(a_{4}, a_{3}\right)_{\gamma}$ and $\left(a_{3}, a_{2}\right)_{\gamma}$. Therefore, if we consider a small flow box $B$ centered at $\left[a_{1}, a_{2}\right]_{f}$ and replace the original $\left[a_{1}, a_{2}\right]_{\gamma}$ by a new segment contained in $B$ and having exactly one tangency point with $\nabla f^{\perp}$ at a point $a_{2}^{\prime}$ close to $a_{2}$, as suggested in figure 3.3 we will obtain a new segment that has less number of tangency points with $\nabla f^{\perp}$ than the original $\gamma$.

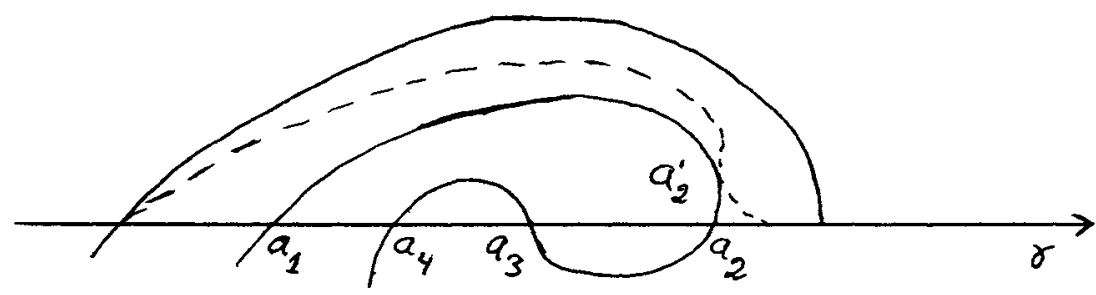

FIG. 3.3

(6) There are three consecutive points $a_{1}, a_{2}$ and $a_{3}$ of a positive half-trajectory of $\nabla f^{\perp}$ such that $\gamma \cap\left[a_{1}, a_{3}\right]_{f}=\left[a_{3}, a_{2}\right]_{\gamma} \cap\left[a_{1}, a_{3}\right]_{f}=$ $\left\{a_{1}, a_{2}, a_{3}\right\}$.

In this situation, to obtain a curve with fewer tangency points with $\nabla f^{\perp}$ we may proceed as in the case just described.

After a finite number of steps we shall find a curve $\gamma$ as required in the lemma.

LEMMA 3.3. - Let $\gamma$ be either as in Lemma 3.2 or having the least possible number of tangency points with $\nabla f^{\perp}$. Then there are numbers

$$
r_{0}=0 \leq l_{1}<r_{1} \leq l_{2}<r_{2} \leq \cdots \leq l_{n}<r_{n} \leq 1=l_{n+1}
$$

satisfying (a) and (b) of Proposition 3.1.

Proof. - Let

$$
0<t_{1}<t_{2}<\cdots<t_{n}<1
$$

be all the real numbers at which $\gamma$ is tangent to $\nabla f^{\perp}$. Given $i$, there exist $r_{i}>t_{i}$ and a continuous family $\left\{L_{s}^{i}: s \in\left(t_{i}, r_{i}\right)\right\}$ of arc of trajectories 
determined by meeting $\gamma$ exactly at their endpoints and by having $\left\{\gamma\left(t_{i}\right)\right\}$ as its limiting set as $s \rightarrow t_{i}$. The assumptions on $\gamma$ imply that if $r_{i}$ is taken to be the greatest possible number, then there must exists $l_{i}$ such that $\gamma\left(\left[l_{i}, r_{i}\right]\right)$ is the compact edge of a half-Reeb component.

It is not difficult to see that, thanks to Lemma 3.2, all the conclusions of this lemma are easily obtained.

LEMMA 3.4. - Let $\gamma$ be as in Lemma 3.2. Then no smooth curve connecting $p$ and $q$ can have fewer points of tangency with $\nabla f^{\perp}$ than $\gamma$. Moreover, if $\gamma^{*}:[0,1] \rightarrow\left[F_{p}, F_{q}\right]$ is another curve having this property of $\gamma$, then the pair $\left(\gamma, \gamma^{*}\right)$ satisfies $(c)$ of Proposition 3.1.

Proof. - As $(a)-(b)$ of Proposition 3.1 have already been proved, we have numbers $r_{i}^{*}, t_{i}^{*}$ and $l_{i}^{*}$ as in the preceding lemma.

Observe that if $s \in\left\{l_{1}, r_{1}, l_{2}, r_{2}, \cdots, r_{n}, l_{n}\right\} \backslash\{0,1\}$ then, as $p$ and $q$ are in different components of $\mathfrak{R}^{2} \backslash \mathfrak{F}_{\gamma(\mathfrak{s})}$, the curve $\gamma^{*}$ has to meet $F_{\gamma(s)}$. Let $\tilde{l}_{i}$ and $\tilde{r}_{i}$ be the points in $[0,1]$ such that $\gamma^{*}\left(\tilde{l}_{i}\right) \in F_{\gamma\left(l_{i}\right)}$ and $\gamma^{*}\left(\tilde{r}_{i}\right) \in F_{\gamma\left(r_{i}\right)}$.

One of the edges of $C_{i}$, with its given orientation, crosses $\gamma^{*}$ from the left side of $\gamma^{*}$ to its right one while the other edge does it in the opposed direction. This implies that $\gamma^{*}\left(\left[\tilde{l}_{i}, \tilde{r}_{i}\right]\right)$ must be tangent to $\nabla f^{\perp}$ somewhere. Therefore, $\gamma^{*}$ must have the same number of points of tangency with $\nabla f^{\perp}$ as $\gamma$.

Now we claim that $\gamma^{*}\left(\left[\tilde{r}_{i}, \tilde{l}_{i+1}\right]\right)$ must be contained in $T_{i}$. In fact, otherwise it would cross some trajectory $F_{m} \notin\left\{F_{\gamma^{*}\left(\tilde{r}_{i}\right)}, F_{\gamma^{*}\left(\tilde{l}_{i+1}\right)}\right\}$ belonging to the boundary of $T_{i}$. As $F_{m}$ separates the plane, $\gamma^{*}\left(\left[\tilde{r}_{i}, \tilde{l}_{i+1}\right]\right)$ would meet $F_{m}$ at least at two points and so it would contain a tangency point with $\nabla f^{\perp}$. This would contradict the minimizing property of $\gamma^{*}$. Therefore, $\gamma^{*}\left(\left[\tilde{r}_{i}, \tilde{l}_{i+1}\right]\right)$ is contained in $T_{i}, \tilde{r}_{i}=r_{i}^{*}, \tilde{l}_{i+1}=l_{i+1}^{*}$ and so $T_{i}^{*}=T_{i}$ proceeding in this way, the proof of this lemma can easily be completed.

\section{THE SETS $\Sigma$ AND $\Sigma_{1}$}

Let $p, q \in \mathfrak{R}^{2}$ be such that $X(p)=X(q)=0$ and let $\left[F_{p}, F_{q}\right]$ be the closure of the connected component of $\mathfrak{R}^{2} \backslash\left(\mathfrak{F}_{\mathfrak{p}} \cup \mathfrak{F}_{\mathfrak{q}}\right)$ whose boundary is $F_{p} \cup F_{q}$. We shall denote by $\Sigma(X, p, q)$ the set of $\theta \in \Re$ such that no transversal region of $\left[F_{p}(\theta), F_{q}(\theta)\right]$ reduces to a single trajectory of $\nabla f_{\theta}^{\perp}$. Let $\Sigma_{1}(X, p, q)=\mathfrak{R} \backslash \Sigma(X, p, q)$. It is clear that $\Sigma_{1}\left(X_{\theta}, H_{\theta}(p), H_{\theta}(q)\right)=$ $\Sigma_{1}(X, p, q)-\theta=\left\{t \in \mathfrak{R}: t+\theta \in \Sigma_{1}(X, p, q)\right\}$. Therefore, we shall only study the case $0 \in \Sigma_{1}(X, p, q)$. Let $\gamma:[0,1] \rightarrow\left[F_{p}, F_{q}\right]$ be a smooth 
curve connecting $\gamma(0)=p$ with $\gamma(1)=q$ and having the least possible number of tangency points with $\nabla f^{\perp}$. Suppose that $\gamma$ has generic contact with $\nabla f^{\perp}$. Let

$$
r_{0}=0 \leq l_{1}<r_{1} \leq l_{2}<r_{2} \leq \cdots \leq l_{n}<r_{n} \leq 1=l_{n+1},
$$

$T_{i}$ and $C_{i}$ be the real numbers, transversal regions and half-Reeb components, respectively, associated to $\left(\left[F_{p}, F_{q}\right], \gamma\right)$ as in Proposition 3.1. We shall say that the sequence

$$
\beta=\left(T_{i}, C_{i+1}, C_{i+2}, \cdots, C_{i+k}, T_{i+k}\right)
$$

with length $k \geq 1$, is a block of $\left(\left[F_{p}, F_{q}\right], \gamma\right)$ if:

(i) Each of $T_{i+1}, T_{i+2}, \cdots, T_{i+k-1}$ is reduced to a single trajectory.

(ii) Either both $T_{i}$ and $T_{i+k}$ are not reduced to a single trajectory (in which case $\beta$ will be called of type $(T, C, \cdots, C, T)$ ) or $T_{i}=T_{0}=F_{p}$ and $T_{i+k}$ is not reduced to a single trajectory ( in which case $\beta$ will be called of type $[C, \cdots, C, T)$ ) or else $T_{n}=T_{i+k}=F_{q}$ and $T_{i}$ is not reduced to a single trajectory ( in which case $\beta$ will be called of type $(T, C, \cdots, C]$ ).

When a half-Reeb component $C_{i+j}$ is on the left of $\gamma$ (resp. on the right of $\gamma$ ), it will denoted by $\cap_{i+j}$ (resp. $\cup_{i+j}$ ) or even simply as $\cap$ (resp. $\cup$ ). According as its length is an even or an odd number and also according as $C_{i+1}$ is on the left or on the right of $\gamma$, a sequence of type $(T, C, \cdots, C, T)$ is from one of the following four subtypes:

$$
\begin{aligned}
& (T, \cup, \cdots, \cap, T) \\
& (T, \cup, \cdots, \cup, T) \\
& (T, \cap, \cdots, \cup, T) \\
& (T, \cap, \cdots, \cap, T) .
\end{aligned}
$$

Similarly, there are four subtypes of each one of the sequences $[C, \cdots, C, T)$ and $(T, C, \cdots, C]$.

Under these conditions we have the following

Proposition 4.1. - Suppose that $0 \in \Sigma_{1}(X, p, q)$ and extend $\gamma$, transversally to $\nabla f^{\perp}$, to an interval $\left[-\varepsilon_{1}, 1+\varepsilon_{1}\right]$. There exists $\varepsilon>0$ and continuous functions

$$
L_{i}, R_{i}: \quad[-\varepsilon, \varepsilon] \rightarrow\left[-\varepsilon_{1}, 1+\varepsilon_{1}\right]
$$

with $L_{i}(0)=l_{i}, R_{i}(0)=r_{i}$, such that if

$$
\beta=\left(T_{i}, C_{i+1}, \cdots, C_{i+k}, T_{i+k}\right)
$$

is a block of $\left(\left[F_{p}, F_{q}\right], \gamma\right)$, then, for $\theta \in(-\varepsilon, \varepsilon)$, it can be constructed a smooth curve $\lambda_{\theta}:[0,1] \rightarrow\left[F_{p}(\theta), F_{q}(\theta)\right]$, with $\lambda_{0}=\gamma$, connecting $H_{\theta}(p)$ 
with $H_{\theta}(q)$, having the least possible number of points of tangency with $\nabla f_{\theta}^{\perp}$ and, in such a way, that one of the twelve statements, $(4.1,1)-(4.1,12)$, of figure 4.1, is satisfied. Each line of such figure corresponds to a pair of conclusions. The way of interpreting each line is the same in all cases. The figures 4.1,1-4.1,12 are related to the statements 4.1,1-4.1,12 of figure 4.1.

Proceed to explain the diagram

$$
(T, \bar{T}, T) \rightarrow(T, \overline{\cap, \cdots, \cup}, T)
$$

which appears in the left side of the first line of figure 4.1. By putting $\theta$ and suffixes in evidence this diagram becomes

$$
\left(T_{i}(\theta), \overline{T(\theta)}, T_{i+k}(\theta)\right) \rightarrow\left(T_{i}, \overline{\Pi_{i+1}}, \cdots, \cup_{i+k}, T_{i+k}\right)
$$

where $\beta=\left(T_{i}, \cap_{i+1}, \cdots, \cup_{i+k}, T_{i+k}\right)$ is in the right side because the conclusions refer to values $\theta<0$. In this case the following is satisfied

(a) $T_{i}(\theta), T(\theta)$ and $T_{i+k}(\theta)$ consist of trajectories that belong to a transversal region of $\left[F_{p}(\theta), F_{q}(\theta)\right]$ and $T(\theta)$ is always a connected set not reduced to a single trajectory.

(b) There are no lines on top of $T_{i}(\theta)$ and $T_{i}$ (resp. $T_{i+k}(\theta)$ and $T_{i+k}$ ) because $\lambda_{\theta}$ and $H_{\theta} \circ \gamma$ coincide in $\left[R_{i}(\theta), L_{i+1}(\theta)\right]$ (resp. $\left.\left[R_{i+k}(\theta), L_{i+k+1}(\theta)\right]\right)$ and $T_{i}(\theta)$ is made of all trajectories of $\nabla f_{\theta}^{\perp}$ that meet $H_{\theta} \circ \gamma\left(\left[R_{i}(\theta), L_{i+1}(\theta)\right]\right)\left(\right.$ resp. $H_{\theta} \circ \gamma\left(\left[R_{i+k}(\theta), L_{i+k+1}(\theta)\right]\right)$ ).

(c) As $\cap_{i+1}, \cdots, \cup_{i+k}$ is not an empty sequence and there are lines on top of $T(\theta)$ and $\cap_{i+1}, \cdots, \cup_{i+k}$, the curve $\lambda_{\theta}$, restricted to $\left[L_{i+1}(\theta), R_{i+k}(\theta)\right]$, cannot taken to be close to $H_{\theta} \circ \gamma$. Also, the trajectories that meet $H_{\theta} \circ \gamma\left(\left[L_{i+1}(\theta), R_{i+k}(\theta)\right]\right)$ form $T(\theta)$. Moreover, $\gamma\left(\left[l_{i+1}, r_{i+k}\right]\right)$ is made up of the compact edges of $\cap_{i+1}, \cdots, \cup_{i+k}$.

In the same way, the right part of the first line of figure 4.1

$$
(T, \cap, \overline{\cup, \cdots, \cap}, \cup, T) \rightarrow(T, \cap, \bar{T}, \cup, T)
$$

refers to values $\theta>0$ because $\beta=(T, \cap, \cup, \cdots, \cap, \cup, T)$ is on the left side. The meaning of this diagram can be deduced in the same way as that of the case above. However, there is a new situation when $k=2$ and so $\bar{U}, \cdots, \cap$ represents an empty sequence. In this particular case $\lambda_{\theta}$ coincides with $H_{\theta} \circ \gamma$ in $\left[R_{i}(\theta), L_{i+k+1}(\theta)\right]$.

There is no new situation in all remaining cases. There is a special case in the third (resp. fourth) line when $k=1$. In this case the block is stable and the diagram could also be written as

$$
(T, \cap, T) \rightarrow(T, \cap, T) \rightarrow(T, \cap, T) .
$$

Vol. $12, n^{\circ} 6-1995$ 


$$
\text { Type } \quad(T, C, \cdots, C, T)
$$

$4.1,1$

$$
(T, \bar{T}, T) \rightarrow(T, \overline{\cap, \cdots, \cup}, T)=(T . \cap . \bar{\cup} \cdots, \bar{\cap}, \cup, T) \rightarrow(T, \cap, \bar{T}, \cup, T)
$$

$4.1,2$

$$
(T, \cup, \bar{T}, \cap, T) \rightarrow(T, \cup, \overline{\cap, \cdots, \cup}, \cap, T)=(T, \bar{U} \cdots, \Pi, T) \rightarrow(T, \bar{T}, T)
$$

$4.1,3$

$$
(T, \bar{T}, \cap, T) \rightarrow(T, \overline{\cap, \cdots, \cup}, \cap, T)=(T . \cap, \bar{\cup}, \cdots, \cap, T) \rightarrow(T, \cap, \bar{T}, T)
$$

$4.1,4$

$$
\begin{gathered}
(T, \cup, \bar{T}, T) \rightarrow(T, \cup, \overline{\cap, \cdots, \cup} . T)=(T, \bar{\cup}, \cdots, \cap, \cup, T) \rightarrow(T, \bar{T}, \cup, T) \\
\text { Type } \quad[C . \cdots, C . T)
\end{gathered}
$$

$4.1,5$

$$
[\bar{T}, T) \rightarrow[\bar{\cap}, \cdots, \cup, T)=(\bar{\cap} \cdots, \cap . \cup . T) \rightarrow[\bar{T}, \cup, T)
$$

$4.1,6$

$$
[\bar{T}, \cap, T) \rightarrow[\overline{\cup, \cdots, \cup}, \cap, T)=[\bar{\cup} \cdots, \cap, T) \rightarrow[\bar{T}, T)
$$

$4.1,7$

$$
[\bar{T}, \cap, T) \rightarrow[\overline{\cap, \cdots, \cup}, \cap, T)=\overline{\cap, \cdots, \cap}, T) \rightarrow[\bar{T}, T)
$$

$4.1,8$

$$
[\bar{T}, T) \rightarrow \overline{\cup, \cdots, \cup}, T)=\overline{\cup, \cdots \cdot \cap}, \cup . T) \rightarrow[\bar{T}, \cup, T)
$$

$$
\text { Type } \quad(T, C, \cdots, C]
$$

$4.1,9$

$$
(T, \bar{T}] \rightarrow(T, \overline{\cap, \cdots, \cup})=(T, \cap, \bar{U} \cdot \cdots, \cup] \rightarrow(T, \cap, \bar{T}]
$$

$4.1,10$

$$
(T, \cup, \bar{T}] \rightarrow(T, \cup, \overline{\cap, \cdots, \cap}]=(T . \bar{U} \cdots, \bar{\cap}] \rightarrow(T, \bar{T})
$$

$4.1,11$

$$
(T, \bar{T}] \rightarrow(T, \overline{\cap, \cdots, \cap}]=(T, \cap, \overline{U, \cdots, \cap]} \rightarrow(T, \cap, \bar{T})
$$

$4.1,12$

$$
(T, \cup, \bar{T}] \rightarrow(T, \cup, \overline{\cap, \cdots, \cup}]=(T, \bar{\cup}, \cdots, \cup) \rightarrow(T, \bar{T}]
$$

Fig. 4.1

The proof of Proposition 4.1 follows immediately from Lemmas 4.2-4.7.

The proof of the following lemma is a direct corollary of Lemma 2.5 . 
Type $\quad(T, \cap, \ldots, \cup, T)$
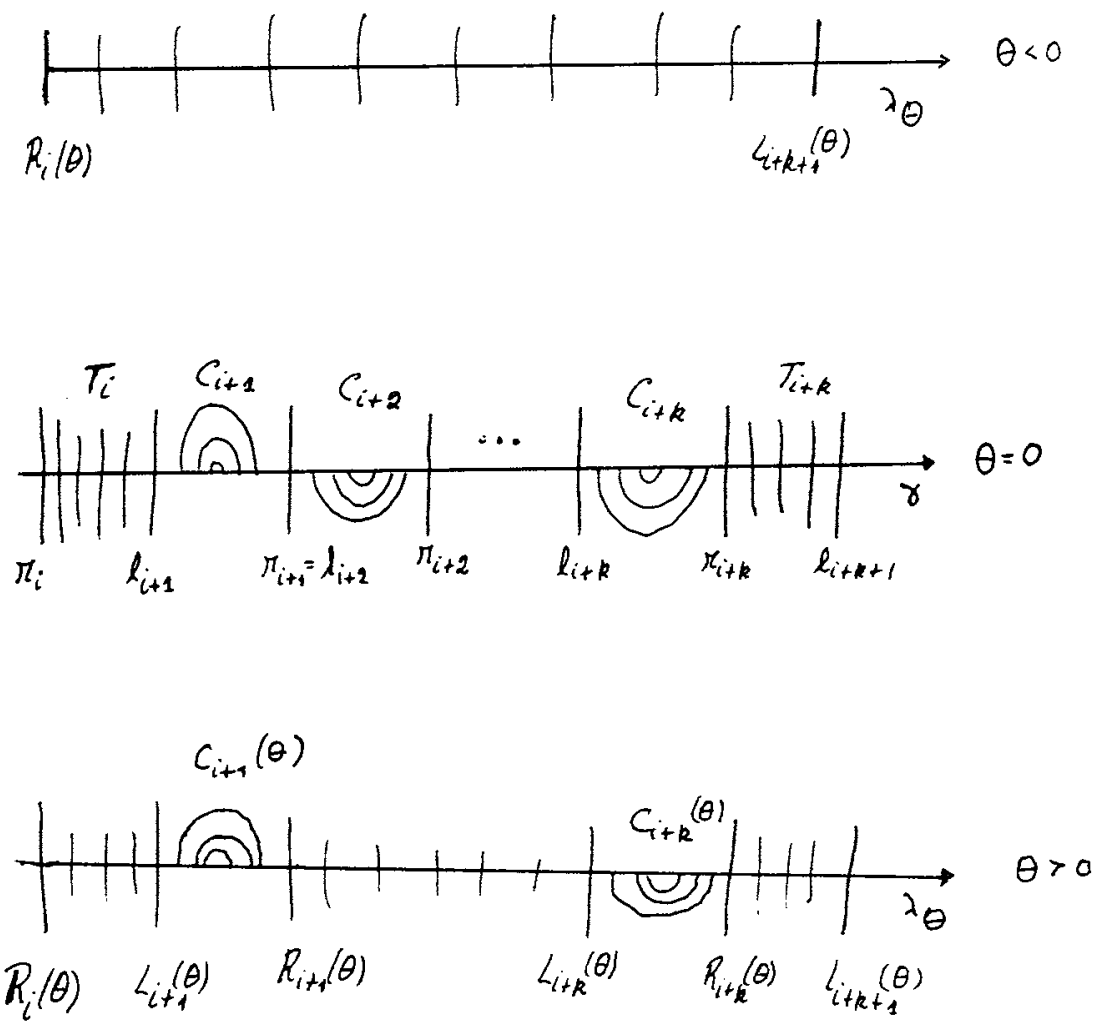

FIG. 4.1,1,a

Lemma 4.2. - If $\varepsilon>0$ is small enough, there are continuous monotone functions

$$
L_{i}, R_{i}: \quad[-\varepsilon, \varepsilon] \rightarrow\left[-\varepsilon_{1}, 1+\varepsilon_{1}\right]
$$

such that, for all $i \in\{1,2, \cdots, n\}, H_{\theta} \circ \gamma\left(\left[L_{i}(\theta), R_{i}(\theta)\right]\right)$ is the compact edge of a half-Reeb component $C_{i}(\theta)$ of $\nabla f^{\perp}$, associated to $H_{\theta} \circ \gamma$, in the sense of Lemma 2.5.

Lemma 4.3. - Given $\varepsilon>0$ small, for all $\theta \in(-\varepsilon, \varepsilon),\left[F_{p}(\theta), F_{q}(\theta)\right]$ contains at least two transversal regions.

Proof. - As $p$ and $q$ are singularities of $X, H_{\theta}(p)$ and $H_{\theta}(q)$ will be singularities of $X_{\theta}$. Because the rest of the argument will depend only on this fact and not on the particular $\theta$, we shall only consider $\left[F_{p}, F_{q}\right]$.

Assume first that $\left[F_{p}, F_{q}\right]$ has no transversal region and that $C_{1}$ is on the left of $\gamma$. This implies, by Proposition 2.1, that if $i$ is an odd number (resp. 

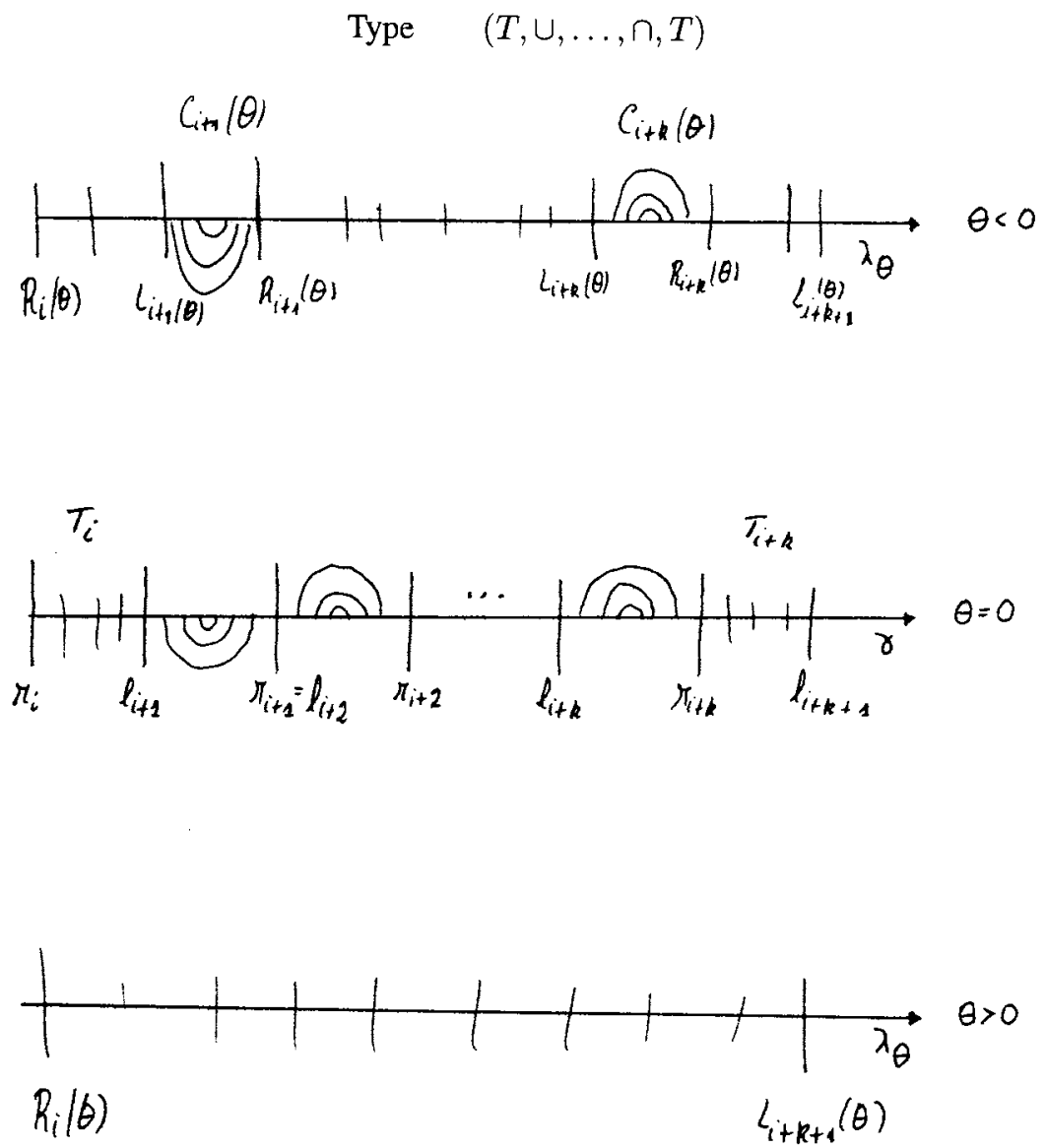

Fig. 4.1,2

even number), $C_{i}$ is on the left (resp. on the right) of $\gamma$. Suppose moreover that $\nabla f$ points inward $C_{1}$ along its non-compact edges and so that they are $F_{\gamma\left(l_{1}\right)}^{+}$and $F_{\gamma\left(r_{1}\right)}^{-}$. As $\operatorname{Det}(D X)>0$ everywhere, $g$ is strictly increasing along trajectories of the vector field $\nabla f^{\perp}$; therefore, $g\left(\gamma\left(l_{1}\right)\right)<g\left(\gamma\left(r_{1}\right)\right)$. Similarly, $g\left(\gamma\left(r_{1}\right)\right)=g\left(\gamma\left(l_{2}\right)\right)<g\left(\gamma\left(r_{2}\right)\right)$. Proceeding in this way we shall find that $g(p)<g(q)$ which is a contradiction. By using the same argument in all the other possibilities, we may conclude that $\left[F_{p}, F_{q}\right]$ must have at least one transversal region.

Now if we assume that $\left[F_{p}, F_{q}\right]$ has exactly one transversal region, by the same argument above, we would conclude that $f(p) \neq f(q)$ which would be again a contradiction. 
Type $\quad(T, \cup, \ldots, \cup, T)$
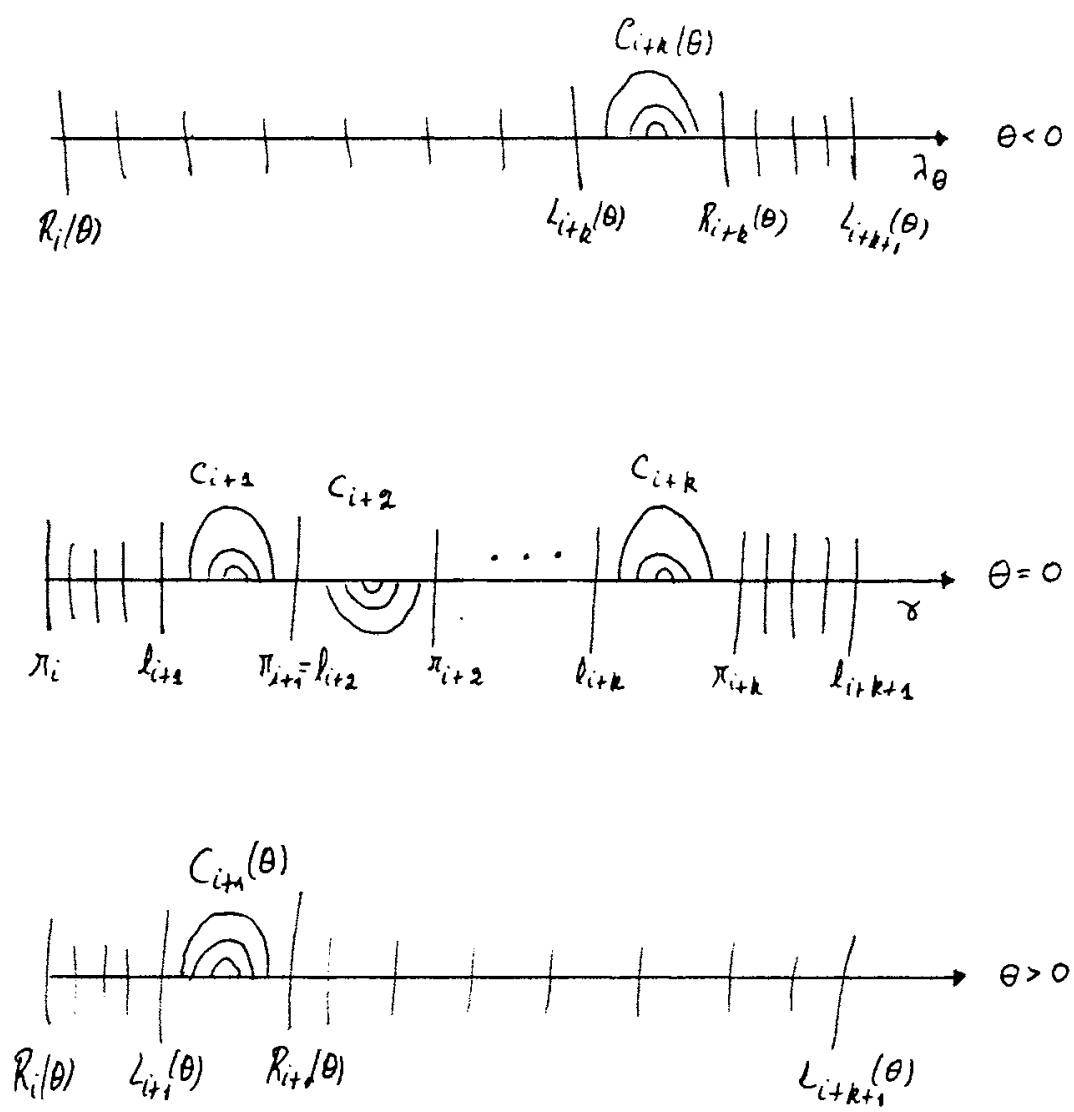

FIG. $4.1,3$

LEMMA 4.4. - Let $i \in\{0,1, \cdots, n\}$ be such that $r_{i}<l_{i+1}$. If $\varepsilon>0$ is small enough then, for all $\theta \in(-\varepsilon, \varepsilon), H_{\theta} \circ \gamma\left(\left[R_{i}(\theta), L_{i+1}(\theta)\right]\right)$ is contained in a transversal region of $\left[F_{p}(\theta), F_{q}(\theta)\right]$.

Proof. - Fix $\theta \in(-\varepsilon, \varepsilon)$. If we assumed by contradiction that there existed an arc of trajectory $\left[a_{1}, a_{2}\right]_{f_{\theta}}$ (of $\nabla f_{\theta}^{\perp}$ ) meeting $H_{\theta} \circ \gamma$ exactly at its endpoints with $a_{1} \in H_{\theta} \circ \gamma\left(\left[R_{i}(\theta), L_{i+1}(\theta)\right]\right)$, this would imply the existence of a half-Reeb component associated to $H_{\theta} \circ \gamma$ (in the context of Lemmas 2.5 and 4.2 ) and containing $\left[a_{1}, a_{2}\right]_{f_{\theta}}$. This contradiction with Lemma 4.2 proves the lemma.

LEMMA 4.5. - Let $i \in\{1,2, \cdots, n-1\}$. Suppose that $l_{i}<r_{i}=l_{i+1}<r_{i+1}$ and that $\varepsilon>0$ is very small. 

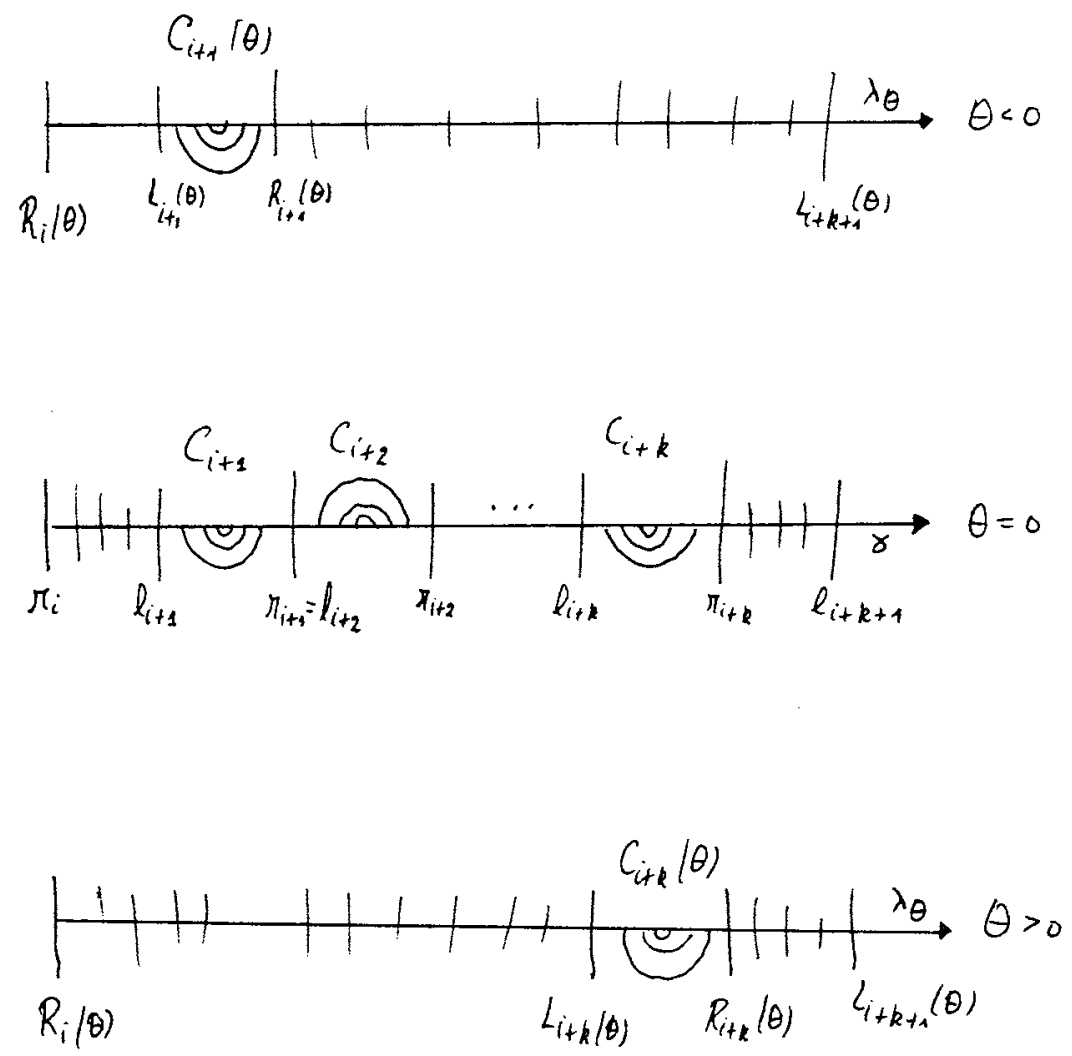

FIG. $4.1,4$

(a) If the half-Reeb component $C_{i}$ (having $\gamma\left(\left[l_{i}, r_{i}\right]\right)$ as its compact edge) is on the left of $\gamma$ then, for $\theta \in(0, \varepsilon), H_{\theta} \circ \gamma\left(\left[R_{i}(\theta), L_{i+1}(\theta)\right]\right)$ is contained in a transversal region of $\left[F_{p}(\theta), F_{q}(\theta)\right]$. See fig. 4.2,a

(b) If the half-Reeb component $C_{i}$ (having $\gamma\left(\left[l_{i}, r_{i}\right]\right)$ as its compact edge) is on the right of $\gamma$ then, for $\theta \in(-\varepsilon, 0), H_{\theta} \circ \gamma\left(\left[R_{i}(\theta), L_{i+1}(\theta)\right]\right)$ is contained in a transversal region of $\left[F_{p}(\theta), F_{q}(\theta)\right]$ and for, $\theta \in$ $(0, \varepsilon), H_{\theta} \circ \gamma\left(\left[L_{i}(\theta), R_{i+1}(\theta)\right]\right)$ is contained in a transversal region of $\left[F_{p}(\theta), F_{q}(\theta)\right]$.

Proof. - Let us prove the item (a) when $\theta \in(-\varepsilon, 0)$. In this case, $L_{i+1}(\theta)<R_{i}(\theta)$ and we may construct a smooth curve $\lambda_{\theta}$, as suggested in figure 4.2 ,a, contained in $\left[F_{p}(\theta), F_{q}(\theta)\right]$, that coincides with $H_{\theta} \circ \gamma$ in the complement of $\left[L_{i}(\theta), R_{i+1}(\theta)\right]$ and that, when restricted to $\left[L_{i}(\theta), R_{i+1}(\theta)\right]$, is transversal to $\nabla f_{\theta}^{\perp}$. Let us assume, by contradiction, 

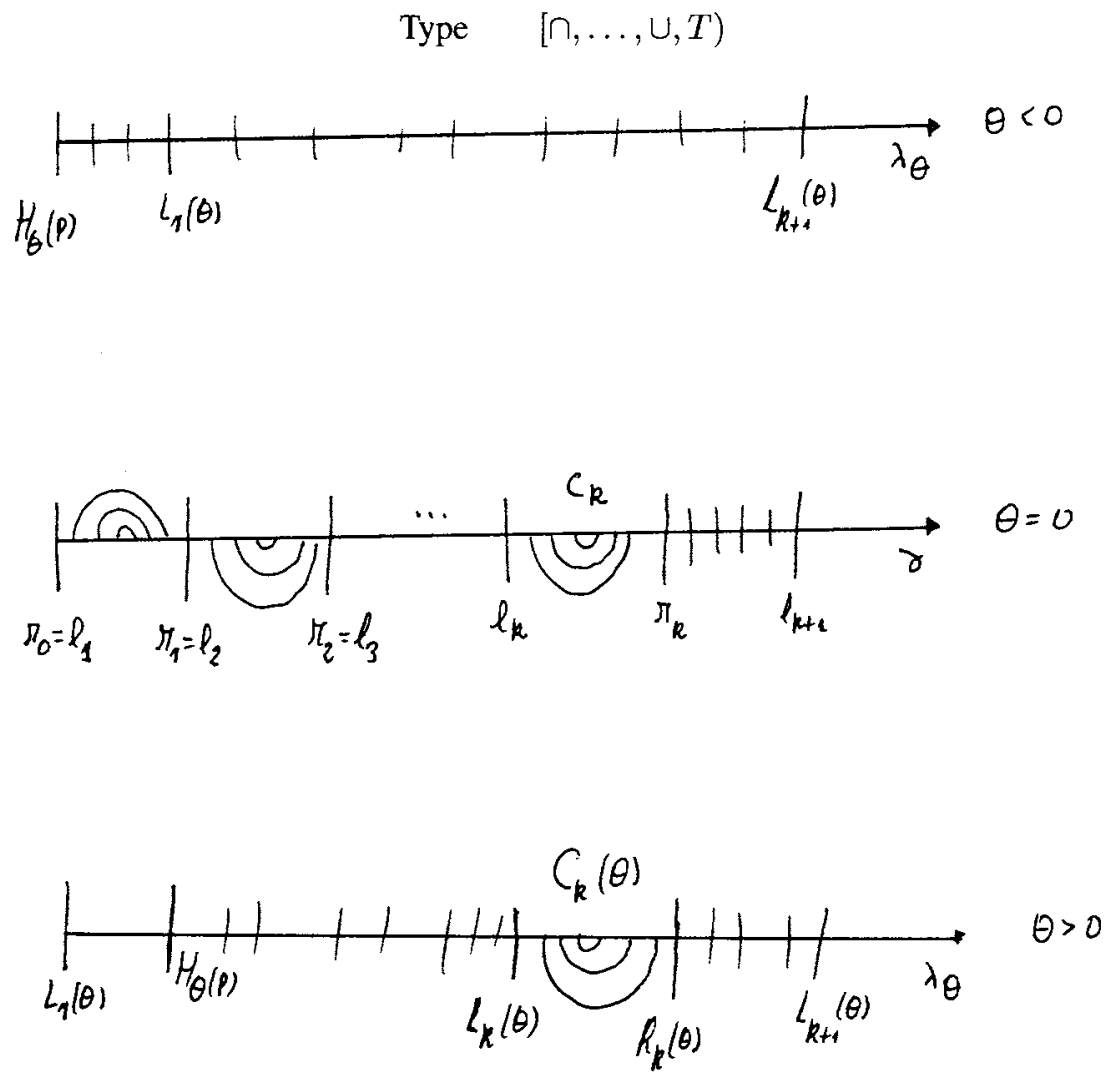

FIG. $4.1,5$

that there exists an arc of trajectory $\left[a_{1}, a_{2}\right]_{f_{\theta}}$ (of $\nabla f_{\theta}^{\perp}$ ) meeting $\lambda_{\theta}$ exactly at its endpoints with $a_{1} \in \lambda_{\theta}\left(\left[L_{i}(\theta), R_{i+1}(\theta)\right]\right)$. By the required transversality, $a_{2}$ cannot belong to $\lambda_{\theta}\left(\left[L_{i}(\theta), R_{i+1}(\theta)\right]\right)$. In particular $a_{2}$ belongs to $H_{\theta} \circ \gamma\left([0,1] \backslash\left[L_{i}(\theta), R_{i+1}(\theta)\right]\right)$. By the orbit structure of $\nabla f_{\theta}$, there must exist an arc of trajectory of $\nabla f_{\theta}$ of the form $\left[b_{1}, a_{2}\right]_{f_{\theta}}$ meeting $H_{\theta} \circ \gamma$ exactly at its endpoints with $b_{1} \in H_{\theta} \circ \gamma\left(\left[L_{i}(\theta), R_{i+1}(\theta)\right]\right)$. This implies the existence of a half-Reeb component associated to $H_{\theta} \circ \gamma$ (in the context of lemmas 2.5 and 4.2) and containing $\left[b_{1}, a_{2}\right]_{f_{\theta}}$. This contradiction with Lemma 4.2 proves this lemma.

Lemma 4.6. - Suppose that $r_{0}=l_{1}$ and that $\varepsilon>0$ is very small.

(a) If the half-Reeb component $C_{1}$ (having $\gamma\left(\left[l_{1}, r_{1}\right]\right)$ as its compact edge) is on the left of $\gamma$ then, there is a continuous function $\sigma:[0, \varepsilon) \rightarrow\left[0, r_{1}\right]$ such that $\sigma(0)=r_{1}, 0<\sigma(\theta) \leq R_{1}(\theta)$, and $H_{\theta}(p)$ and $H_{\theta} \circ \gamma(\sigma(\theta))$ are the endpoints of the subarc of $F_{p}(\theta)$ that is on the left of $\gamma$ and that meets Vol. $12, n^{\circ}$ 6-1995. 
Type $\quad[\cup, \ldots, \cap, T)$
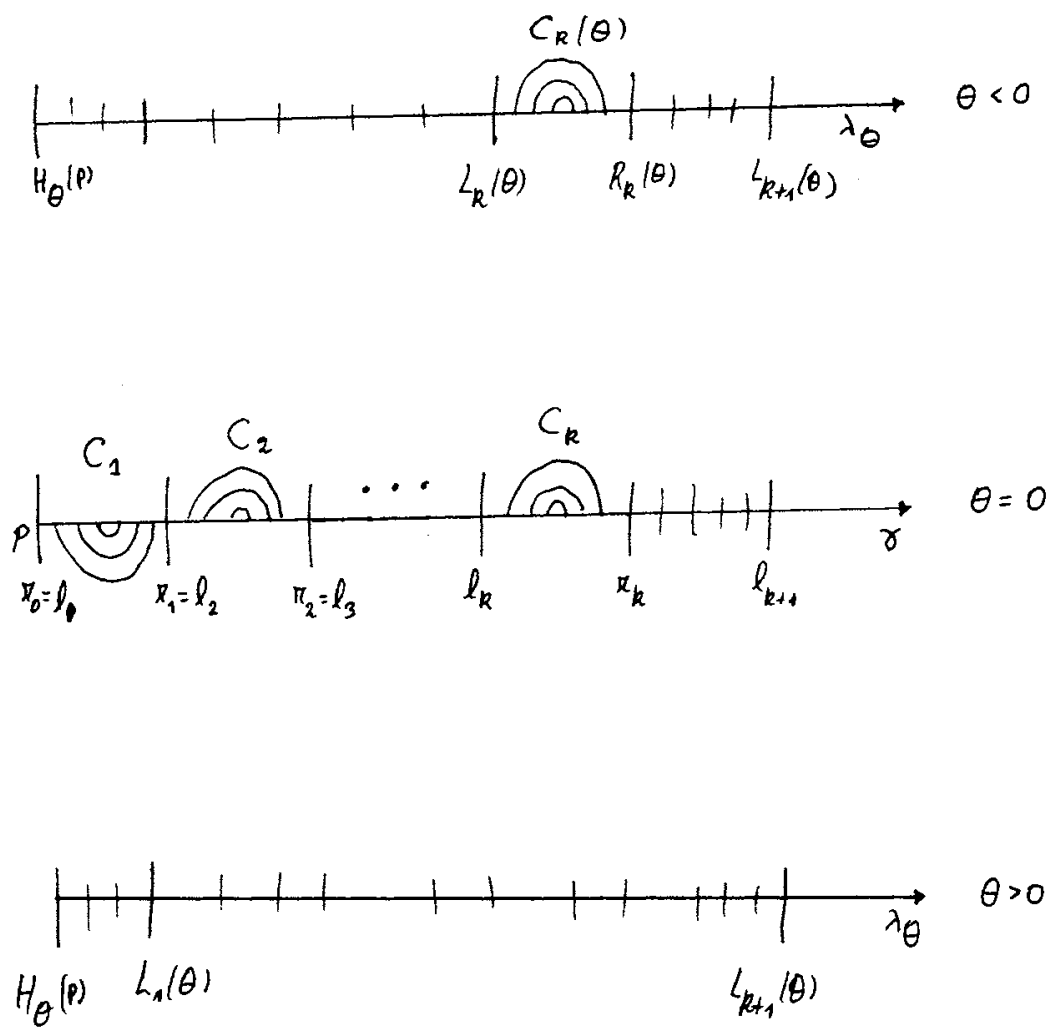

FIG. 4.1,6

$H_{\theta} \circ \gamma$ exactly at its endpoints. Also, $H_{\theta} \circ \gamma\left(\left[\sigma(\theta), R_{1}(\theta)\right]\right.$ is contained in a transversal region of $\left[F_{p}(\theta), F_{q}(\theta)\right]$. Moreover, for, $\theta \in(-\varepsilon, 0)$, $H_{\theta} \circ \gamma\left(\left[0, L_{1}(\theta)\right]\right)$ is contained in a transversal region of $\left[F_{p}(\theta), F_{q}(\theta)\right]$. See fig. 4.3,a.

(b) If the half-Reeb component $C_{1}$ (having $\gamma\left(\left[l_{1}, r_{1}\right]\right)$ as its compact edge) is on the right of $\gamma$ then, there is a continuous function $\sigma:(-\varepsilon, 0] \rightarrow\left[0, r_{1}\right]$ such that $\sigma(0)=r_{1}, 0<\sigma(\theta) \leq R_{1}(\theta)$, and $H_{\theta}(p)$ and $H_{\theta} \circ \gamma(\sigma(\theta))$ are the endpoints of the subarc of $F_{p}(\theta)$ that is on the right of $\gamma$ and that meets $H_{\theta} \circ \gamma$ exactly at its endpoints. Also, $\gamma\left(\left[\sigma(\theta), R_{1}(\theta)\right]\right.$ is contained in a transversal region of $\left[F_{p}(\theta), F_{q}(\theta)\right]$. Moreover, for, $\theta \in[0, \varepsilon), H_{\theta} \circ \gamma\left(\left[0, L_{1}(\theta)\right]\right)$ is contained in a transversal region of $\left[F_{p}(\theta), F_{q}(\theta)\right]$. See fig. 4.3,b.

Proof. - Let us prove the item $(a)$ when $\theta \in(0, \varepsilon)$. In this case, $0>L_{1}(\theta)$ and so, by Lemma 2.2 and Lemma $2.5,(d)$, there must exist a function 

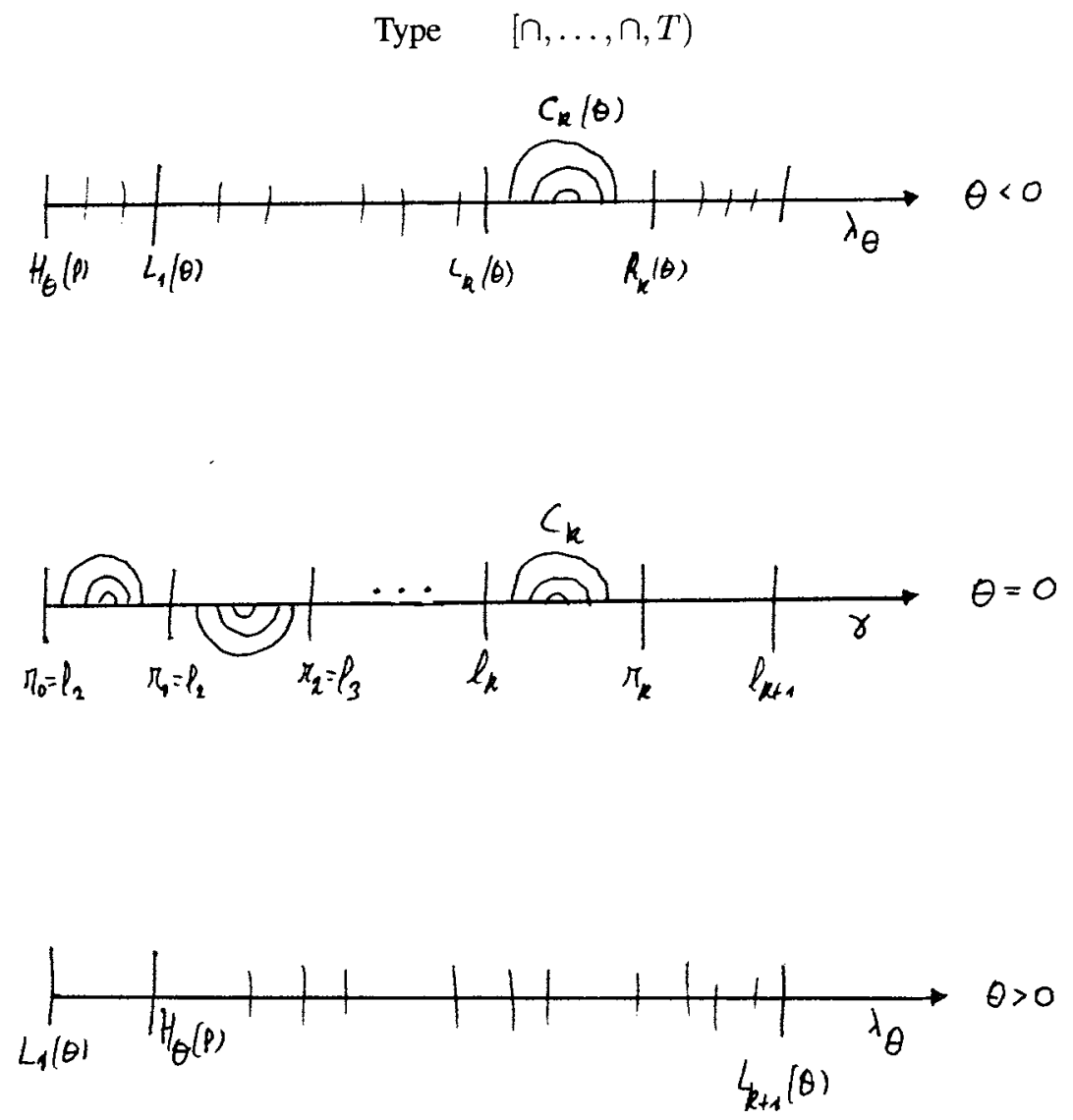

FIG. $4.1,7$

$\sigma$ as required. The proof of the remainder of $(a)$ is similar to that of Lemma 4.4: We construct a smooth curve $\lambda_{\theta}:[0,1] \rightarrow\left[F_{p}(\theta), F_{q}(\theta)\right]$, as drawn in dotted lines in figure 4.3, a, that coincides with $\left.H_{\theta} \circ \gamma\right|_{[0,1]}$ in the complement of $\left[0, R_{1}(\theta)\right]$ and, when restricted to $\left[0, R_{1}(\theta)\right]$, is transversal to $\nabla f_{\theta}^{\dagger}$. Using a similar argument to that of Lemma 4.4, we may show that trajectories of $\nabla f_{\theta}^{\perp}$ can meet $\lambda_{\theta}\left(\left[0, R_{1}(\theta)\right]\right)$ at most once. This implies, by Proposition 3.1, our claim.

Lemma 4.7. - Suppose that $r_{n}=l_{n+1}$ and that $\varepsilon>0$ is very small.

(a) If the half-Reeb component $C_{n}$ (having $\gamma\left(\left[l_{n}, r_{n}\right]\right)$ as its compact edge) is on the right of $\gamma$ then, there is a continuous function $\sigma:[0, \varepsilon) \rightarrow\left[l_{n}, 1\right]$ such that $\sigma(0)=l_{n}, 1>\sigma(\theta) \geq L_{n}(\theta)$, and $H_{\theta}(q)$ and $H_{\theta} \circ \gamma(\sigma(\theta))$ are the endpoints of the subarc of $F_{q}(\theta)$ that is on the right of $\gamma$ and that meets $H_{\theta} \circ \gamma$ exactly at its endpoints. Also, $H_{\theta} \circ \gamma\left(\left[L_{n}(\theta), \sigma(\theta)\right]\right.$ is contained Vol. $12, n^{\circ} 6-1995$. 
Type $\quad[\cup, \ldots, \cup, T)$
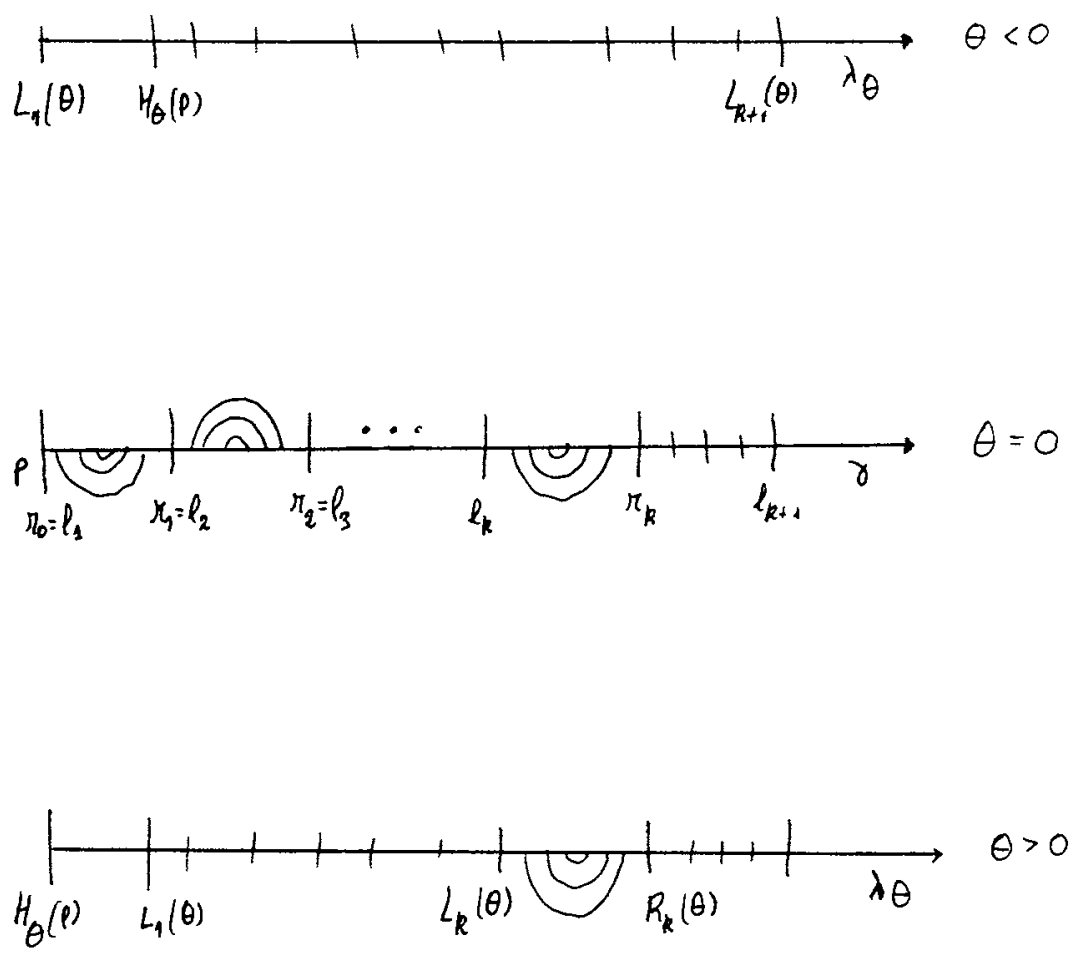

FIG. $4.1,8$

in a transversal region of $\left[F_{p}(\theta), F_{q}(\theta)\right]$. Moreover, for, $\theta \in(-\varepsilon, 0)$, $H_{\theta} \circ \gamma\left(\left[R_{n}(\theta), 1\right]\right)$ is contained in a transversal region of $\left[F_{p}(\theta), F_{q}(\theta)\right]$.

(b) If the half-Reeb component $R_{n}$ (having $\gamma\left(\left[l_{n}, r_{n}\right]\right)$ as its compact edge) is on the left of $\gamma$ then, there is a continuous function $\sigma:(-\varepsilon, 0] \rightarrow\left[l_{n}, 1\right]$ such that $\sigma(0)=l_{n}, 1>\sigma(\theta) \geq L_{n}(\theta)$, and $H_{\theta}(p)$ and $H_{\theta} \circ \gamma(\sigma(\theta))$ are the endpoints of the subarc of $F_{p}(\theta)$ that is on the left of $\gamma$ and that meets $H_{\theta} \circ \gamma$ exactly at its endpoints. Also, $\gamma\left(\left[L_{n}(\theta), \sigma(\theta)\right]\right.$ is contained in a transversal region of $\left[F_{p}(\theta), F_{q}(\theta)\right]$. Moreover, for, $\theta \in[0, \varepsilon), H_{\theta} \circ \gamma\left(\left[R_{n}(\theta), 1\right]\right)$ is contained in a transversal region of $\left[F_{p}(\theta), F_{q}(\theta)\right]$.

COROLlaRY 4.8. - (a) $\Sigma_{1}(X, p, q)$, modulo $2 \pi$, consists of a finite set of points.

(b) If $\theta_{1}, \theta_{2}$ belong to the same connected component of $\Sigma(X, p, q) \backslash$ $\Sigma_{1}(X, p, q)$, then the cannonical decompositions of $\left(\left[F_{p}\left(\theta_{1}\right), F_{q}\left(\theta_{1}\right)\right], \lambda_{\theta_{1}}\right)$ and $\left(\left[F_{p}\left(\theta_{2}\right), F_{q}\left(\theta_{2}\right)\right], \lambda_{\theta_{2}}\right)$, as given by Proposition 3.1, are essentially the same: 

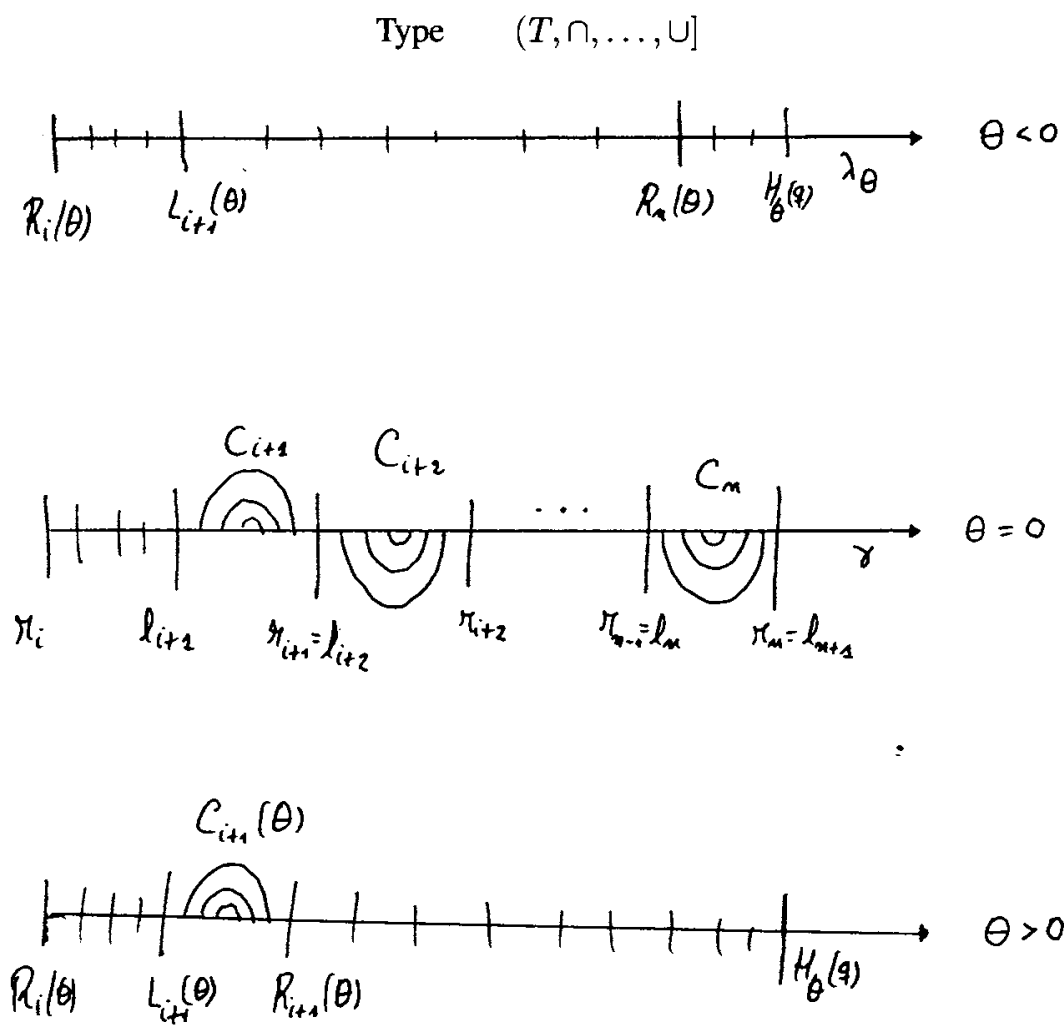

FIG. $4.1,9$

b1) They have the same number of transversal regions and half-Reeb components,

b2) every transversal region of both is not reduced to a single orbit, and

b3) provided that half-Reeb components of both are ordered as in Proposition 3.1, the ones in correspondence are both simultaneously on the right or on the left of the related curve $\lambda_{\theta_{i}}$.

Remark 4.9. - The elements of $\Sigma(X, p, q)$ behave as the stable systems and those of $\Sigma_{1}(x, p, q)$ are the bifurcating ones.

The proof of Theorem A, done in Section 5, will be obtained by studying the behaviour of the canonical decomposition of $\left(\left[F_{p}(\theta), F_{q}(\theta)\right], \lambda_{\theta}\right)$, as $\theta$ varies in $R$. 
C. GUTIERREZ
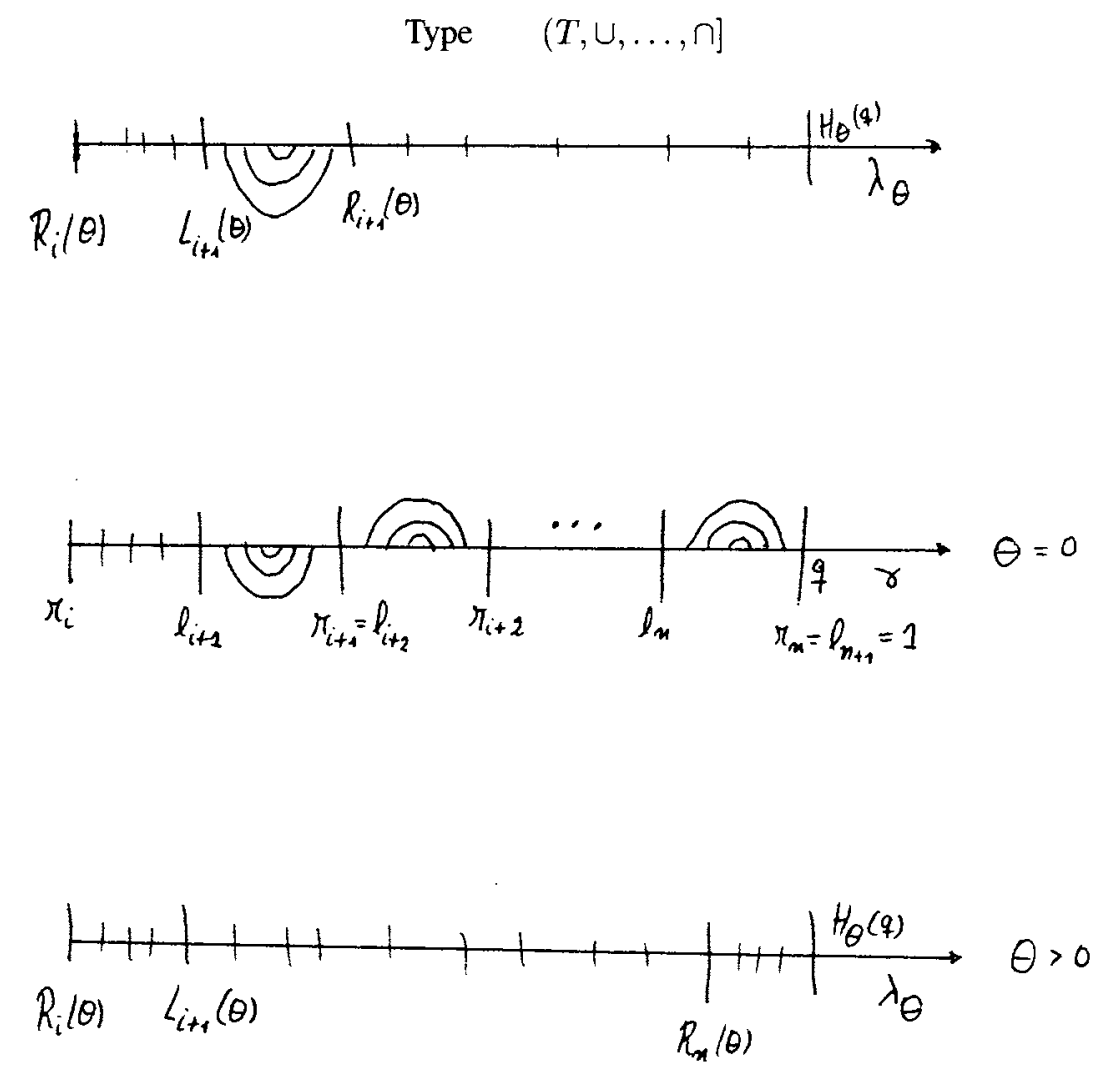

FIG. $4.1,10$

\section{PROOF OF THEOREM A}

THEOREM A. - Any smooth map $X: \mathfrak{R}^{2} \rightarrow \mathfrak{R}^{2}$ satisfying the $\rho$-eigenvalue condition, for some $\rho \in[0, \infty)$, is injective.

Proof. - Suppose by contradiction that $X$ is not injective. Then, by composing $X$ with a translation, it can be assumed that there exists $p, q \in \mathfrak{R}^{2}$ such that $X(p)=X(q)=0$. Let $\gamma:[0,1] \rightarrow\left[F_{p}, F_{q}\right]$ be a smooth curve connecting $\gamma(0)=p$ with $\gamma(1)=q$ and having the least possible number of tangency points with $\nabla f^{\perp}$. Suppose that $\gamma$ has generic contact with $\nabla f^{\perp}$. Let

$$
r_{0}=0 \leq l_{1}<r_{1} \leq l_{2}<r_{2} \leq \cdots \leq l_{n}<r_{n} \leq 1=l_{n+1},
$$

and

$$
\left(T_{0}, C_{1}, T_{1}, C_{2}, T_{2} \cdots, C_{n}, T_{n}\right)
$$


Type $\quad(T, \cap, \ldots, \cap]$
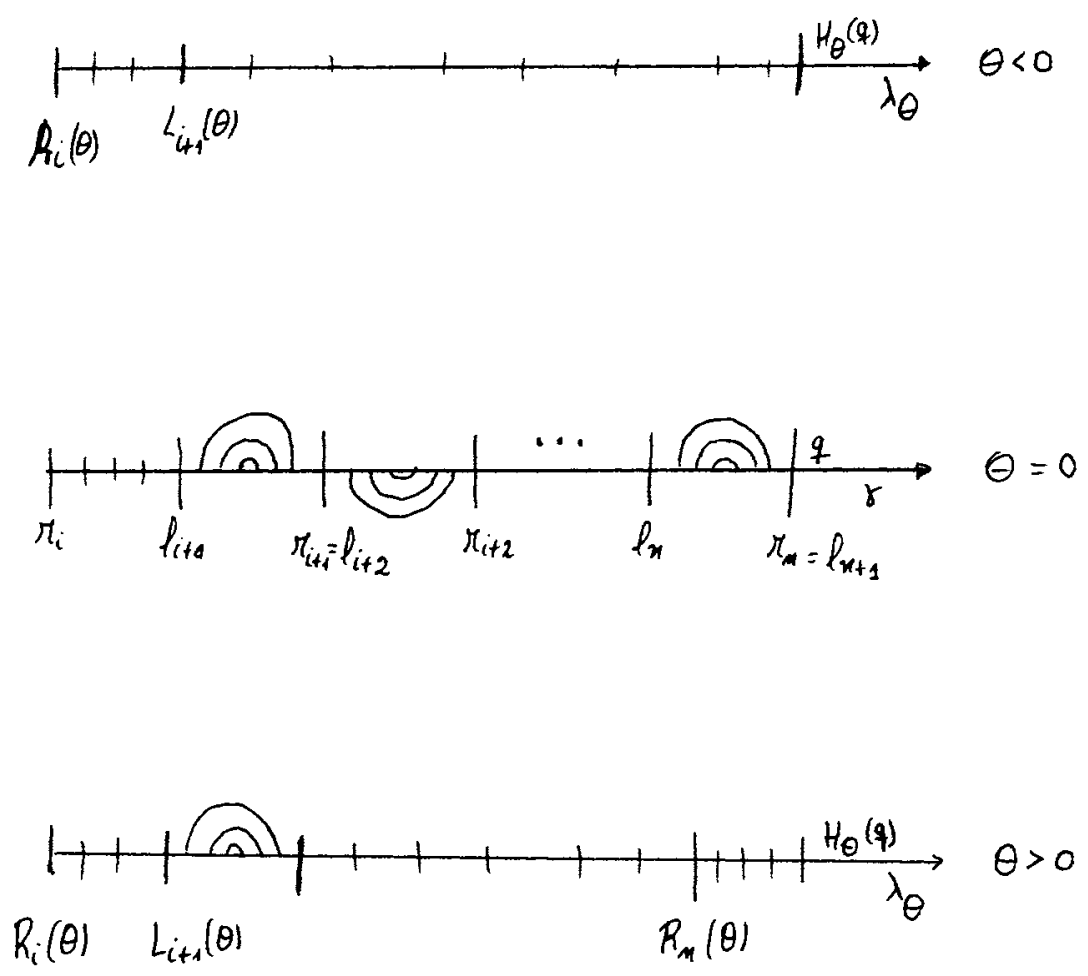

FIG. 4.1,11

be the sequence of real numbers and the sequence made up of transversal regions and half-Reeb components, respectively, associated to $\left(\left[F_{p}, F_{q}\right], \gamma\right)$ as in Proposition 3.1.

Given $\theta \in \mathfrak{R} / 2 \pi \mathbf{Z}$ choose a smooth curve $\lambda_{\theta}:[0,1] \rightarrow\left[F_{p}(\theta), F_{q}(\theta)\right]$ connecting $\lambda_{\theta}(0)=H_{\theta}(p)$ with $\lambda_{\theta}(1)=H_{\theta}(q)$ and having the least possible number of tangency points with $\nabla f_{\theta}^{\perp}$. Suppose that $\lambda_{0}=\gamma$. For all $\theta \in \Re$, let $T_{0}(\theta)$ and $D_{1}(\theta)$ be the first transversal region and half-Reeb component, respectively, associated to $\left(\left[F_{p}(\theta), F_{q}(\theta)\right], \lambda_{\theta}\right)$ as in Proposition 3.1. In particular, $T_{0}(0)=T_{0}$ and $D_{1}(0)=C_{1}$. We shall denote by $B_{1}(\theta)$ the only trajectory of $\nabla f_{\theta}^{\perp}$ that contains $T_{0}(\theta) \cap D_{1}(\theta)$.

By replacing $X$ by $X_{\theta}$ if necessary, we may assume that $T_{0}$ does not consists of a single trajectory.

Let $L_{1}, L_{2}, \cdots, L_{k}$ be $C^{1}$ embedded images of $\mathfrak{R}$ such that each of them separates the plane into two connected components. We say that $\left\{L_{1}, L_{2}, \cdots, L_{k}\right\}$ is a $(p, q)$-separating sequence if, for all $j \in$ Vol. $12, n^{\circ}$ 6-1995. 

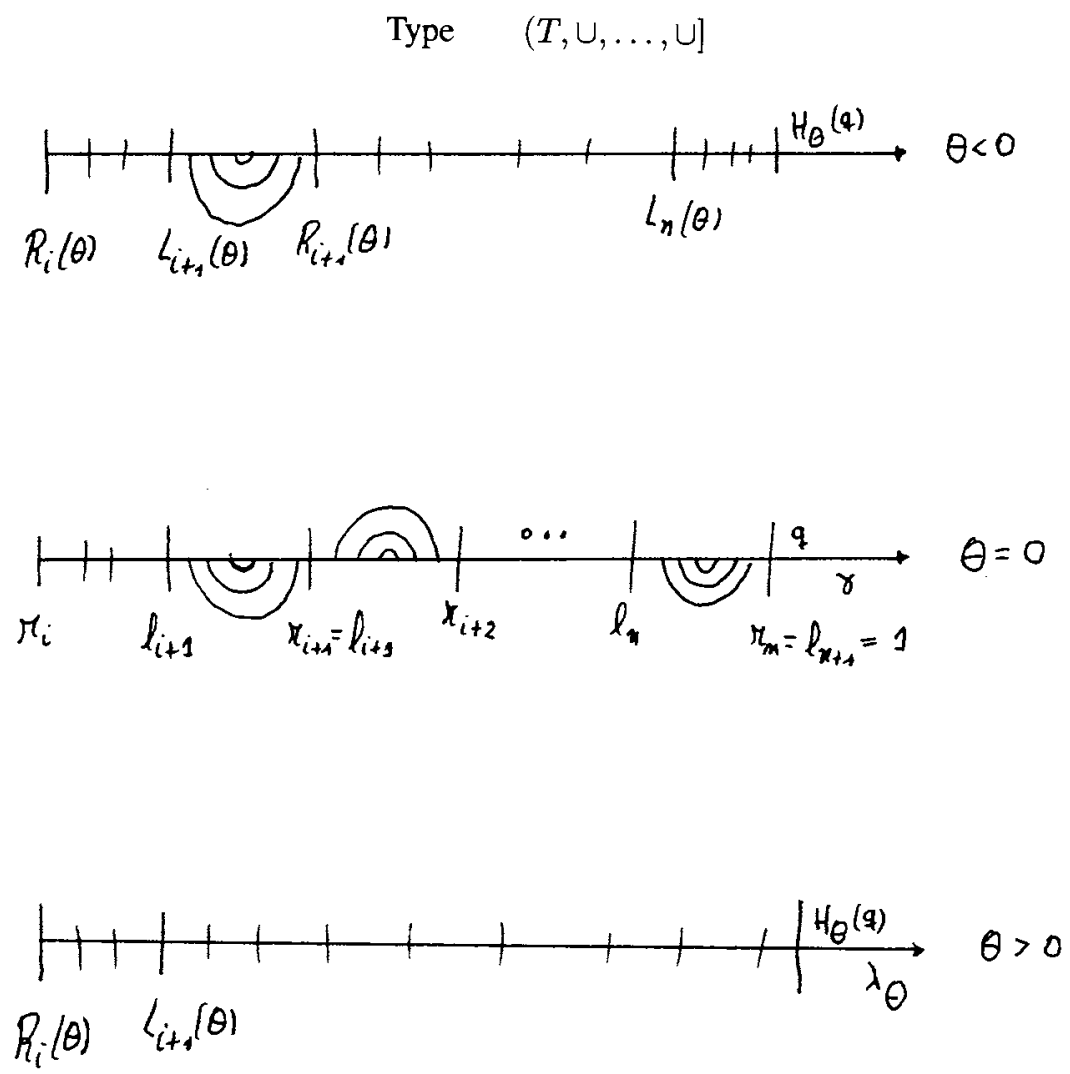

FIG. 4.1,12

$\{1,2, \cdots, k\}$

$$
\{p\} \cup L_{1} \cup \cdots \cup L_{j-1} \quad \text { and } \quad L_{j+1} \cup \cdots \cup L_{k} \cup\{q\}
$$

are contained in different connected components of $\mathfrak{R}^{2} \backslash \mathfrak{L}_{\mathrm{j}}$, where $L_{0}$ and $L_{k+1}$ denote the empty set.

To proceed with the proof we shall need the following:

Lemma 5.1. - Let suppose that $C_{1}$ is on the right of $\gamma$ (resp. on the left of $\gamma$ ). If $\varepsilon>0$ is small enough and $0 \leq \theta<\varepsilon$ (resp. $0 \geq \theta>-\varepsilon$ ), then $\left\{B_{1}(0), H_{\theta}^{-1}\left(B_{1}(\theta)\right)\right\}$ is a $(p, q)$-separating sequence, and also $D_{1}(\theta)$ is on the right of $\lambda_{\theta}$ (resp. on the left of $\lambda_{\theta}$ ).

Proof. - We shall only consider the case in which $C_{1}=\cup_{1}$ is on the right of $\gamma$. 

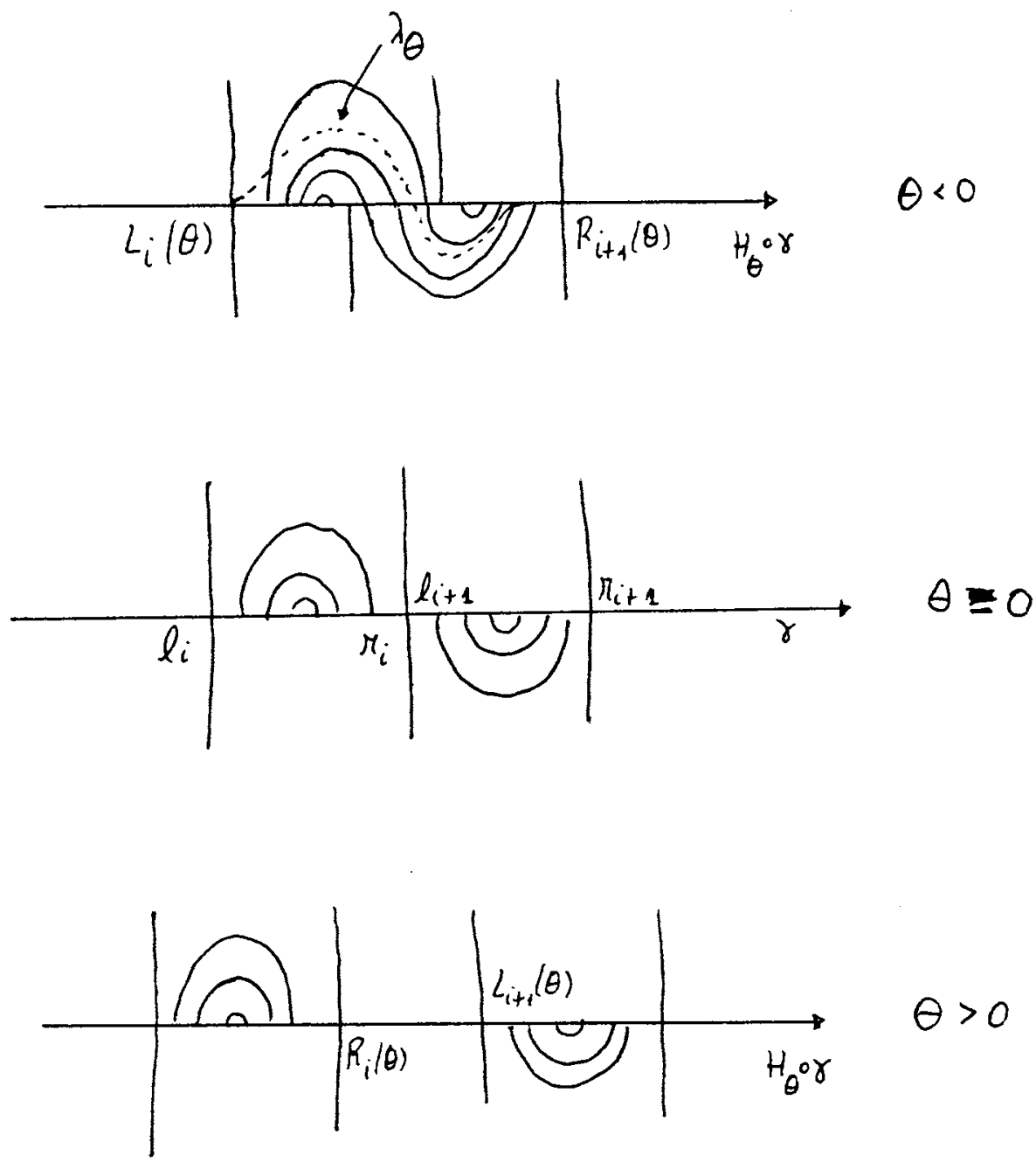

FIG. 4.2,a

Suppose first that $0 \in \Sigma(X, p, q)$. This implies that all the blocks of $\left(\left[F_{p}, F_{q}\right], \gamma\right)$ are of type $(T, C, \cdots, C, T)$ and have length 1 . This means that the blocks are either of the form $(T, \cup, T)$ or $(T, \cap, T)$. Hence, for $\theta$ small, $\lambda_{\theta}$ can be taken to be equal to $H_{\theta} \circ \gamma$. Let $\varepsilon>0, L_{i}(\theta)$ and $R_{i}(\theta)$ be as in Proposition 4.1. It follows from claim 4.1.4 of this proposition (which in this case takes the form $(T, \cup, T) \rightarrow(T, \cup, T) \rightarrow(T, \cup, T))$ and from Lemma 2.5,(d), that $L_{1}(\theta)$ and $R_{1}(\theta)$ are strictly increasing functions of $\theta$ (with $L_{1}(0)=l_{1}, R_{1}(0)=r_{1}$ ), that $D_{1}(\theta)$ is on the right of $\lambda_{\theta}$ and has 

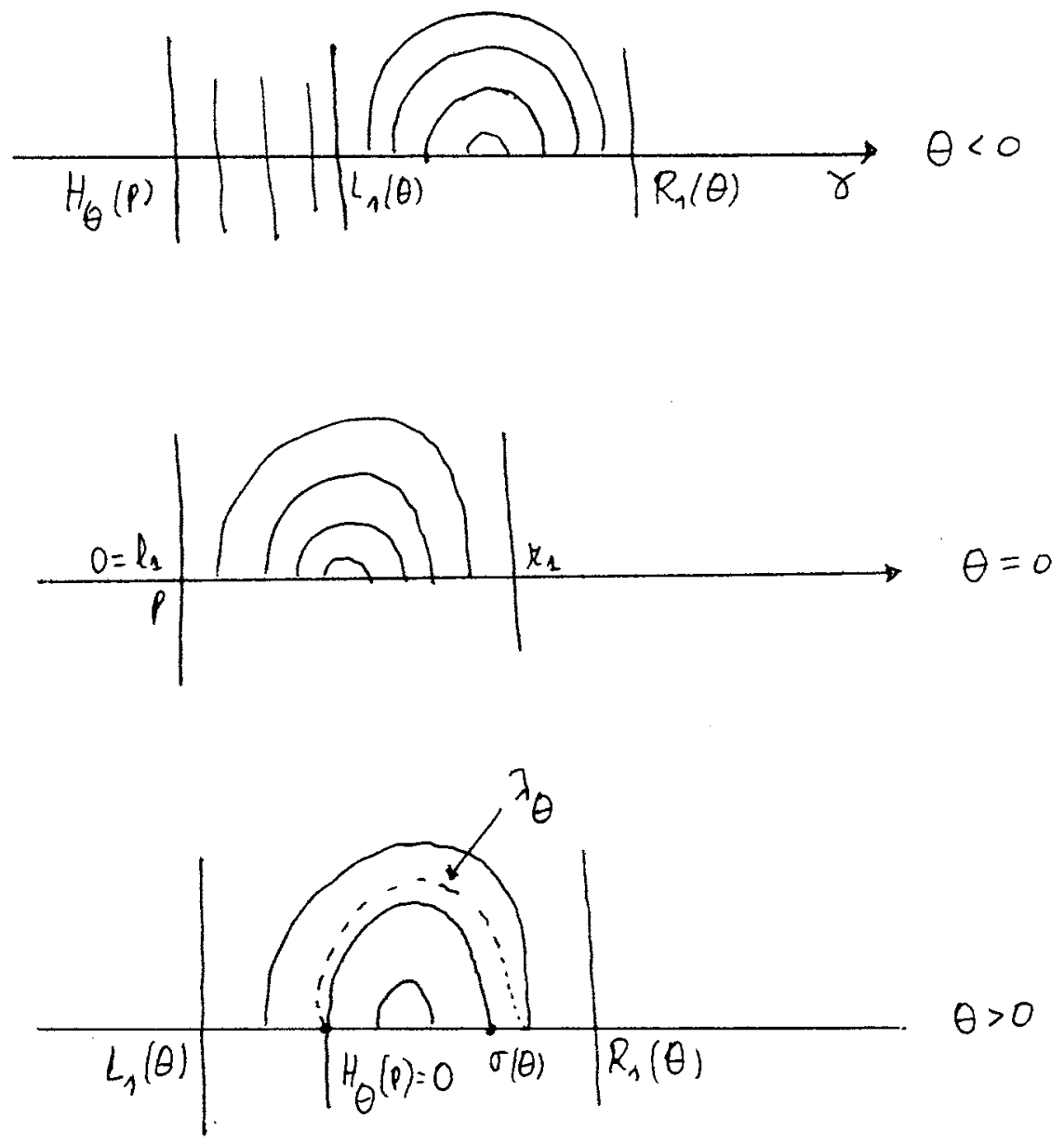

FIG. 4.3,a

$H_{\theta} \circ \gamma\left(\left[L_{1}(\theta), R_{1}(\theta)\right]\right)$ as its compact edge and also that the trajectory of $\nabla f_{\theta}^{\perp}$ passing through $H_{\theta} \circ \gamma\left(L_{1}(\theta)\right)$ is precisely $B_{1}(\theta)$. Given $\theta \in(0, \varepsilon)$, denote by $F_{\gamma\left(t_{1}\right)}(\theta)$ the trajectory of $\nabla f_{\theta}^{\perp}$ passing through $H_{\theta}\left(\gamma\left(t_{1}\right)\right)$. (See fig. 5.1).

It follows from Lemma 2.2 that

(1) $H_{\theta}\left(\left(B_{1}(0)\right)\right.$ is transversal to $\nabla f_{\theta}^{\perp}$ and meets $F_{\gamma\left(t_{1}\right)}(\theta)$ exactly at $H_{\theta}\left(\gamma\left(t_{1}\right)\right)$.

Hence, as the connected component of $F_{\gamma\left(t_{1}\right)}(\theta) \backslash\left\{H_{\theta}\left(\gamma\left(t_{1}\right)\right)\right\}$, contained in the left side of $H_{\theta} \circ \gamma$, is disjoint of $B_{1}(\theta)$, we must also have that the connected component of $H_{\theta}\left(\left(B_{1}(0)\right) \backslash\left\{H_{\theta}\left(\gamma\left(t_{1}\right)\right)\right\}\right.$ contained in the left side of $H_{\theta} \circ \gamma$ is disjoint of $B_{1}(\theta)$. Therefore, as $B_{1}(\theta)$ 

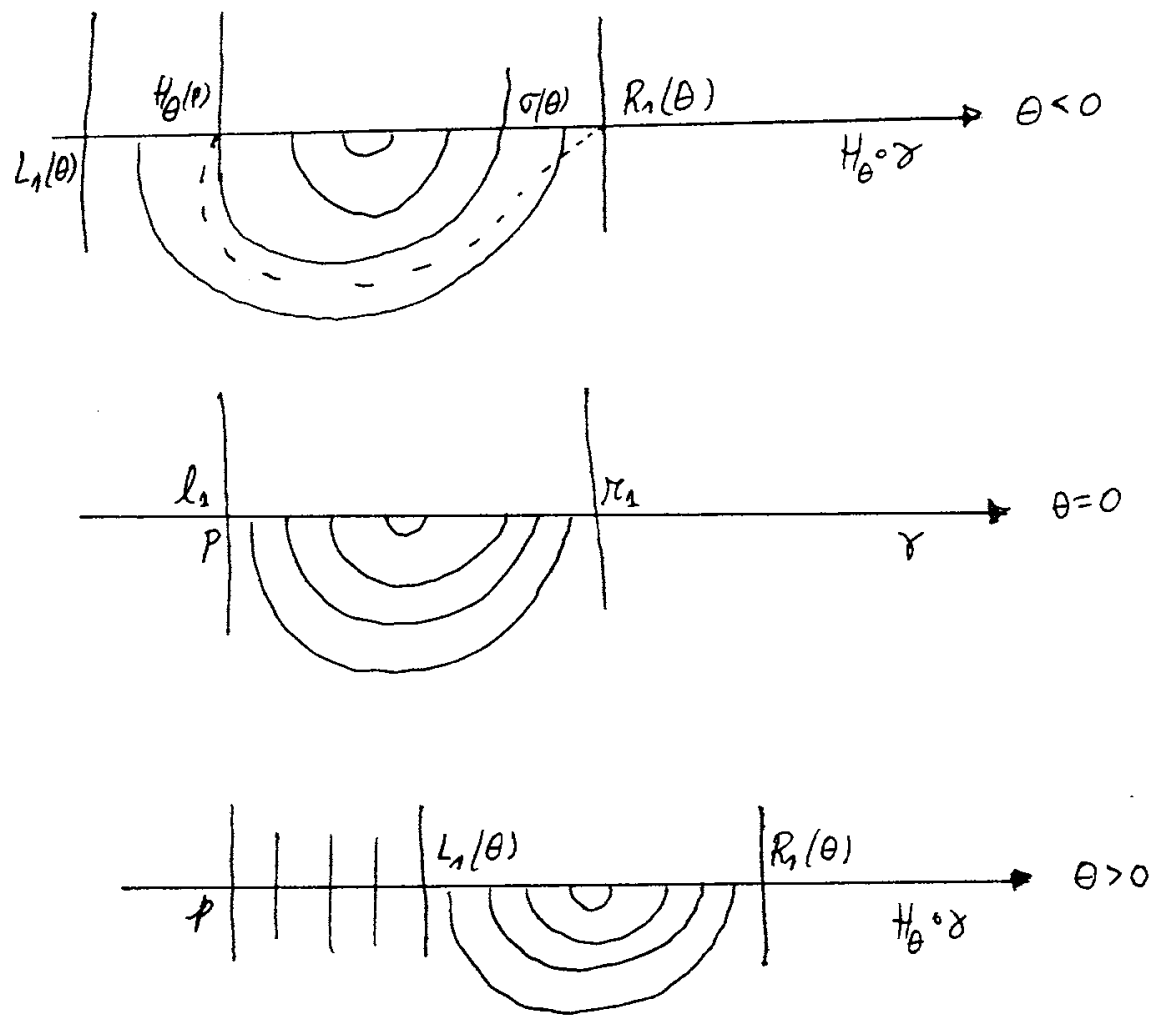

FIG. 4.3,b

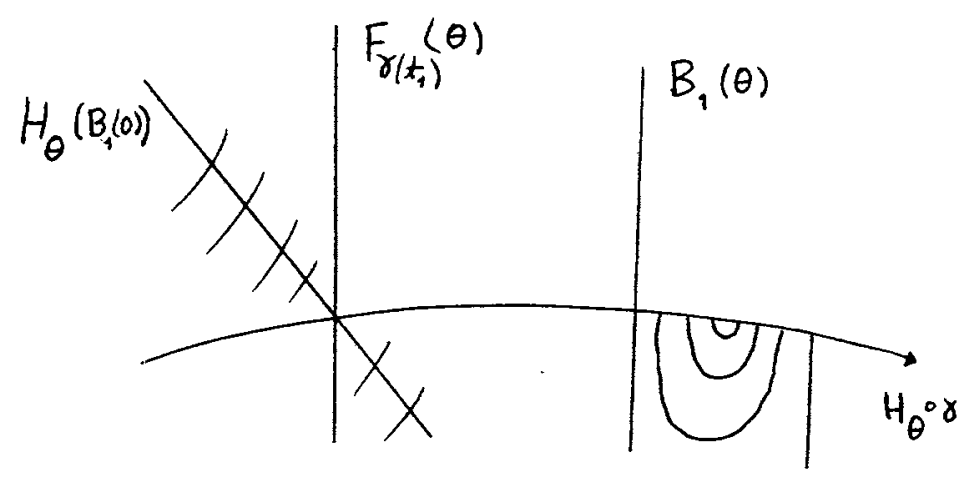

FIG. 5.1

contains the edge of $D_{1}(\theta)$ (which is on the right of $H_{\theta} \circ \gamma$ ) it must be that not only $H_{\theta}\left(\left(B_{1}(0)\right)\right.$ is disjoint of $B_{1}(\theta)$ but also that $\left\{H_{\theta}\left(\left(B_{1}(0)\right), B_{1}(\theta)\right\}\right.$ is a $\left(H_{\theta}(p), H_{\theta}(q)\right)$-separating sequence. This implies Vol. $12, \mathrm{n}^{\circ}$ 6-1995. 
that $\left\{B_{1}(0), H_{\theta}^{-1}\left(B_{1}(\theta)\right)\right\}$ is a $(p, q)$-separating sequence. In the case considered the lemma follows immediately from this.

In what follows, we shall omit the proof of all the statements in which we (implicitly) claim that $\left\{B_{1}(0), H_{\theta}^{-1}\left(B_{1}(\theta)\right)\right\}$ is a $(p, q)$-separating sequence. The reason is that the required arguments are very similar to the one given right above.

Suppose now that $0 \in \Sigma_{1}(X, p, q)$. Let $\beta_{1}, \beta_{2}, \cdots, \beta_{l}$, be the blocks of $\left(\left[F_{p}, F_{q}\right], \gamma\right)$ ordered in the natural way, with $T_{0} \in \beta_{1}$.

It follows from Proposition 4.1 that $\beta_{1}$ is of one of the following subtypes: $(T, \cup, \cdots, \cup, T),(T, \cup, \cdots, \cap, T),(T, \cup, \cdots, \cap]$ and $(T, \cup, \cdots, \cup]$. By Lemma $4.3, \beta_{1}$ cannot be of type $(T, C, \cdots, C]$. If $\beta_{1}$ is of subtype $(T, \cup, \cdots, \cup, T)$ then (by Statement $4.1,4$ of Proposition 4.1 and Lemma $2.5,(d)$ ) we may see that the lemma is true. If $\beta_{1}$ is of subtype $(T, \cup, \cdots, \cap, T)$ then, by Proposition 2.1 , the second block $\beta_{2}$ must be either of type $(T, \cup, \cdots, C, T)$ or of type $(T, \cup, \cdots, C]$, where $C \in\{\cap, \cup\}$.

We claim that $\beta_{2}$ cannot be of type $(T, \cup, \cdots, \cup]$. Otherwise, if we denote by $\beta_{i}(\theta)$, with $i \in\{1,2\}$ and $\beta_{i}(0)=\beta_{i}$, the "block" of $\left(\left[F_{p}(\theta), F_{q}(\theta)\right], \lambda_{\theta}\right)$ emerging from $\beta_{i}$, as $\theta$ varies, in the sense of Statements $4.1,2$ and 4.1,12, of Proposition 4.1, we will have that, for $\theta>0$ small, $\beta_{1}(\theta)=(T, T, T)$ and $\beta_{2}(\theta)=(T, T]$ are both part of transversal regions of $\left[F_{p}(\theta), F_{q}(\theta)\right]$. By the type of $\beta_{2}$, the only blocks of $\left(\left[F_{p}, F_{q}\right], \gamma\right)$ are precisely $\beta_{1}$ and $\beta_{2}$ and therefore $\beta_{1}(\theta)$ and $\beta_{2}(\theta)$, for $\theta$ small, have to form all the blocks of $\left(\left[F_{p}(\theta), F_{q}(\theta)\right], \lambda_{\theta}\right)$. This implies that $\left(\left[F_{p}(\theta), F_{q}(\theta)\right], \lambda_{\theta}\right)$ will have only one transversal region. This contradiction with Lemma 4.3 proves our claim.

In a similar way (see Statement $4.1,10$ of Proposition 4.1 ), $\beta_{2}$ cannot be of type $(T, \cup, \cdots, \cap]$ and so $\beta_{2}$ is either of type $(T, \cup, \cdots, \cup, T)$ or of type $(T, \cup, \cdots, \cap, T)$. If $\beta_{2}$ is of type $(T, \cup, \cdots, \cup, T)$, then the lemma is true. If $\beta_{2}$ is of type $(T, \cup, \cdots, \cap, T)$, then we shall proceed to study the third block an so on. As there can be only finitely many blocks and by the same argument used above to show that $\beta_{2}$ cannot be of type $(T, \cup, \cdots, \cup, T]$, we conclude that all blocks $\beta_{i}$ cannot be of type $(T, \cup, \cdots, \cap, T)$. Hence, we shall eventually find a sequence of consecutive blocks $\beta_{1}, \beta_{2}, \cdots, \beta_{m}$ of type $(T, \cup, \cdots, \cap, T)$ such that $\beta_{m+1}$ is of type $(T, \cup, \cdots, \cup, T)$. Under these conditions and by an argument similar to the case above the lemma follows.

LEMMA 5.2. - Suppose that, for some $\theta \in(-\varepsilon, 0), D_{1}(\theta)$ is on the right of $\lambda_{\theta}$ (resp. on the left of $\lambda_{\theta}$ ). If $\varepsilon>0$ is small enough then, for all $\theta \in(-\varepsilon, 0]$, $D_{1}(\theta)$ is on the right of $\lambda_{\theta}$ (resp. on the left of $\lambda_{\theta}$ ) and $0 \geq \theta>-\varepsilon$ (resp. $0 \leq \theta<\varepsilon$ ) implies that $\left\{H_{\theta}^{-1}\left(B_{1}(\theta)\right), B_{1}(0)\right\}$ is a $(p, q)$-separating sequence. 
Proof. - If $0 \in \Sigma(X, p, q)$, then the proof is similar to that of Lemma 5.1 and will be omitted.

Suppose that $0 \in \Sigma_{1}(X, p, q)$. We shall only consider the case in which for some $\theta \in(-\varepsilon, 0), D_{1}(\theta)$ is on the right of $\lambda_{\theta}$. If $\varepsilon>0$ is small enough, it follows from Corollary 4.8 that, for all $\theta \in(-\varepsilon, 0), \theta \in \Sigma(X, p, q)$. Therefore, using Proposition 3.1 and the same arguments as that of the beginning of the proof of Lemma 5.1,

(1) for all $\theta \in(-\varepsilon, 0), D_{1}(\theta)$ is on the right of $\lambda_{\theta}$.

Let $\beta_{1}, \beta_{2}, \cdots, \beta_{l}$, be the blocks of $\left(\left[F_{p}, F_{q}\right], \gamma\right)$ ordered in the natural way, with $T_{0} \in \beta_{1}$. Take $\varepsilon>0$ so small that all the Statements 4.1,1$4.1,12$ are true when are to be applied to the blocks $\beta_{k}$. It follows from Proposition 4.1 that if a half-Reeb component $C_{i}$ of $\left(\left[F_{p}, F_{q}\right], \gamma\right)$ persists when $\theta \in(-\varepsilon, 0]$ varies, then

(2.1) $\lambda_{\theta}$ restricted to $\left[L_{i}(\theta), R_{i}(\theta)\right]$ coincides with $H_{\theta} \circ \gamma$ and the persisting half-Reeb component $C_{i}(\theta)$ of $\left(\left[F_{p}(\theta), F_{q}(\theta)\right], \lambda_{\theta}\right)$ is precisely the one given by one of the Statements 4.1,1-4.1,12 of the proposition.

(2.2) Every half-Reeb component of $\left(\left[F_{p}(\theta), F_{q}(\theta)\right], \lambda_{\theta}\right)$ is of the form $C_{i}(\theta)$ as described in (2.1).

Hence, there exists $1 \leq j \leq n$ such that, for all $\theta \in(-\varepsilon, 0]$, $C_{j}(\theta)=D_{1}(\theta)$. In particular

(3) $C_{j}$ is on the right of $\gamma$ and belongs to a block $\beta_{i}$ of one of the following subtypes: $(T, \cup, \cdots, \cup, T),(T, \cup, \cdots, \cap, T),(T, \cup, \cdots, \cap]$ and $(T, \cup, \cdots, \cup]$.

Denote by $\beta_{i}(\theta)$, with $\beta_{i}(0)=\beta_{i}$, the "block" of $\left(\left[F_{p}(\theta), F_{q}(\theta)\right], \lambda_{\theta}\right)$ emerging from $\beta_{i}$, as $\theta$ varies, in the sense of Statements 4.1,1-4.1,12 of Proposition 4.1 (for instance, if $\beta_{i}=(T, \cap, \cdots, \cup, T)$ is as in Statement $4.1,1$, then, for all $\theta<0, \beta_{i}(\theta)=(T, T, T)$ and, for $\theta>0$, $\left.\beta_{i}(\theta)=(T, \cap, T, \cup, T)\right)$.

We claim that $i=1$. If we assume by contradiction that $i>1$, then, by (2.1) and (2.2), we will have that $\beta_{i-1}(\theta)$, with $\theta \in(-\varepsilon, 0)$, must be formed by transversal regions only. Therefore, by analyzing the Statements 4.1,1-4.1,12 of Proposition 4.1, $\beta_{i-1}$ can only be of one of the following types:

$$
(T, \cap, \cdots, \cup, T), \quad[\cap, \cdots, \cup, T) \quad \text { and } \quad[\cup, \cdots, \cup, T) \text {. }
$$

However, if we put together $\beta_{i-1}$ and $\beta_{i}$ we shall obtain a contradiction with Proposition 2.1 (see (3)). This proves that $\beta_{i}=\beta_{1}$.

Under these conditions, we conclude from (3) and from Lemma 4.3 that

(4) $\beta_{1}$ is either of type $(T, \cup, \cdots, \cup, T)$ or $(T, \cup, \cdots, \cap, T)$. 
Hence, it follows from Statements 4.1,2 and 4.1,4 of Proposition 4.1 that not only $C_{j}=C_{1}$ but also that the arguments of Lemma 5.1 can be used to finish the proof of this lemma.

End of the proof of Theorem A. - Consider only the case in which $C_{1}$ is on the right. Let $[0, \varphi)$ be the maximal subinterval of $\Re$ such that if $0 \leq \theta<\varphi$ then $\left\{B_{1}(0), H_{\theta}^{-1}\left(B_{1}(\theta)\right)\right\}$ is a $(p, q)$-separating sequence. It follows from Lemmas 5.1 and 5.2 and standard arguments based on the connectedness and compactness of closed intervals that $\varphi=\infty$. More precisely, given an interval $[0, b]$, by its compactness and by Lemmas 5.1 and 5.2, there exists a finite sequence $0=\theta_{0}<\theta_{1}<\cdots<\theta_{l-1}<\theta_{l}=b$ such that, for all $i \in\{0,1, \cdots, l-1\}$,

$$
\left\{B_{1}\left(\theta_{i}\right),\left(H_{\theta_{i+1}-\theta_{i}}\right)^{-1}\left(B_{1}\left(\theta_{i+1}\right)\right)\right\}
$$

is a $\left(H_{\theta_{i}}(p), H_{\theta_{i}}(q)\right)$-separating sequence. Considering the cases $i \in\{0,1\}$, this implies that

$$
\left\{B_{1}(0), H_{\theta_{1}}^{-1}\left(B_{1}\left(\theta_{1}\right)\right), H_{\theta_{2}}^{-1}\left(B_{1}\left(\theta_{2}\right)\right)\right\}
$$

is a $(p, q)$-separating sequence. By induction,

$$
\left\{B_{1}(0), H_{\theta_{1}}^{-1}\left(B_{1}\left(\theta_{1}\right)\right), \cdots, H_{\theta_{l}}^{-1}\left(B_{1}\left(\theta_{l}\right)\right)\right\}
$$

is a $(p, q)$-separating sequence. In particular,

$$
\left\{B_{1}(0), H_{\theta_{l}}^{-1}\left(B_{1}\left(\theta_{l}\right)\right)\right\}
$$

is a $(p, q)$-separating sequence. This proves that $\varphi=\infty$.

However, this is a contradiction because $H_{2 \pi}$ is the identity map and so $\left\{B_{1}(0), H_{2 \pi}^{-1}\left(B_{1}(2 \pi)\right)=B_{1}(0)\right\}$ cannot be a $(p, q)$-separating sequence.

\section{REFERENCES}

[1] M. A. Alzerman, On a problem concerning the stability in the large of dynamical systems., Uspehi Mat. Nauk. N. S., Vol. 4, (4), pp. 187-188.

[2] N. E. Barabanov, On a problem of Kalman., Siberian Mathematical Journal, Vol. 29, (3), 1988, pp. 333-341.

[3] R. FESSLER, A solution of the two dimensional Global Asymptotic Jacobian Stability Conjecture, Preprint. ETH-Zentrum, Switzerland.

[4] A. GASULL, J. LLIBRE and J. SOTOMAYOR, Global asymptotic stability of differential equations in the plane, $J$. diff. Eq., 1989, To appear.

[5] A. GASUlL and J. SoTOMAYOR, On the basin of attraction of dissipative planar vector fields, Lecture Notes in Mathematics. Springer-Verlag. Procc. Coll. Periodic Orbits and Bifurcations. Luminy, 1989, To appear. 
[6] G. GoRNI and G. ZAMPIERI, On the global conjecture for global asymptotic stability, 1990, To appear.

[7] C. GUTTERREZ, Dissipative vector fields on the plane with infinitely many attracting hyperbolic singularities, Bol. Soc. Bras. Mat., Vol. 22, No. 2, 1992, pp. 179-190.

[8] P. HaRTMan, On stability in the large for systems of ordinary differential equations, Can. J. Math., Vol. 13, 1961, pp. 480-492.

[9] P. HaRTMAN, Ordinary differential equations, Sec. Ed. Birkhäuser, 1982.

[10] R. E. Kalman, On Physical and Mathematical mechanisms of instability in nonlinear automatic control systems, Journal of Applied Mechanics Transactions, ASME, Vol. 79, (3), 1957, pp. 553-566.

[11] N. N. KRASOVSKII, Some problems of the stability theory of motion, 1959, In russian. Gosudartv Izdat. Fiz. Math. Lit., Moscow., English translation, Stanford University Press, 1963.

[12] L. Markus and H. Yamabe, Global stability criteria for differential systems, Osaka Math. J., Vol. 12, 1960, pp. 305-317.

[13] G. MeISTERS and O. OLECH, Global Stability, injectivity and the Jacobian Conjecture, To appear in the Procc. of the First World Congress on Nonlinear Analysis held at Tampa, Florida. August, 1992.

[14] G. MeISTERS and O. OLECH, Solution of the global asymptotic stability Jacobian conjecture for the polynomial case, Analyse Mathématique et applications. Contributions en l'honneur de J. L. Lions., Gauthier-Villars, Paris, 1988, pp. 373-381.

[15] C. Olech, On the global stability of an autonomous system on the plane, Cont. to Diff. Eq., Vol. 1, 1963, pp. 389-400.

[16] B. SMITH and F. XAVIER, Injectivity of local diffeomorphisms from nearly spectral conditions, University of Notre Dame, Preprint, 1993.

(Manuscript received April 26, 1993;

revised May, 1994.) 